\title{
A note on tsunamis: their generation and propagation in an ocean of uniform depth
}

\author{
BY JOSEPH L. HAMMACK
}

W.M. Keck Laboratory of Hydraulics and Water Resources, California Institute of Technology, Pasadena

(Received 21 March 1973)

The waves generated in a two-dimensional fluid domain of infinite lateral extent and uniform depth by a deformation of the bounding solid boundary are investigated both theoretically and experimentally. An integral solution is developed for an arbitrary bed displacement (in space and time) on the basis of a linear approximation of the complete (nonlinear) description of wave motion. Experimental and theoretical results are presented for two specific deformations of the bed; the spatial variation of each bed displacement consists of a block section of the bed moving vertically either up or down while the time-displacement history of the block section is varied. The presentation of results is divided into two sections based on two regions of the fluid domain: a generation region in which the bed deformation occurs and a downstream region where the bed position remains stationary for all time. The applicability of the linear approximation in the generation region is investigated both theoretically and experimentally; results are presented which enable certain gross features of the primary wave leaving this region to be determined when the magnitudes of parameters which characterize the bed displacement are known. The results indicate that the primary restriction on the applicability of the linear theory during the bed deformation is that the total amplitude of the bed displacement must remain small compared with the uniform water depth; even this restriction can be relaxed for one type of bed motion.

Wave behaviour in the downstream region of the fluid domain is discussed with emphasis on the gradual growth of nonlinear effects relative to frequency dispersion during propagation and the subsequent breakdown of the linear theory. A method is presented for finding the wave behaviour in the far field of the downstream region, where the effects of nonlinearities and frequency dispersion have become about equal. This method is based on the use of a model equation in the far field (which includes both linear and nonlinear effects in an approximate manner) first used by Peregrine (1966) and more recently advocated by Benjamin, Bona \& Mahony (1972) as a preferable model to the more commonly used equation of Korteweg \& de Vries (1895). An input-output approach is illustrated for the numerical solution of this equation where the input is computed from the linear theory in its region of applicability. Computations are presented and compared with experiment for the case of a positive bed displacement where the net volume of the generated wave is finite and positive; the results demonstrate the 
evolution of a train of solitary waves (solitons) ordered by amplitude followed by a dispersive train of oscillatory waves. The case of a negative bed displacement in which the net wave volume is finite and negative (and the initial wave is negative almost everywhere) is also investigated; the results suggest that only a dispersive train of waves evolves (no solitons) for this case.

\section{Introduction}

Tsunamis are waves generated primarily by submarine earthquakes of shallow focus during which vertical deformations of the sea bed occur. The general structure of tsunamis in the deep ocean is fairly well known at the present time; typical wavelengths are large and wave heights are very small compared with ocean depths. When these waves approach a coastal region where the water depth decreases rapidly, the wave energy is focused by refraction which, combined with shoaling and local resonance effects, may result in significantly increased wave amplitudes. These large waves then strike the shoreline of exposed areas, presenting a major hazard to life and property in heavily populated regions.

Most of the previous analytical investigations of tsunami generation and propagation have been based on a linearized description of wave motion in either a two- or three-dimensional fluid domain of uniform depth. The complexity of the integral solutions developed from the linear theory even for the simplest model of bed deformation prevented many authors from determining detailed wave behaviour, especially near the source region. Using different approximations for these integral solutions and numerical computations, Ichiye $(1950,1958)$, Honda \& Nakamura (1951), Webb (1962) and Kajiura (1963) were able to determine the general wave pattern near the source region for a variety of bed motions in a two-dimensional fluid domain. For very rapid movements of the bed, the water surface displacement initially approximated the shape of the bed deformation and then divided into two wave trains propagating in opposite directions. The maximum amplitude of the largest wave leaving the generation region for these bed motions was found never to exceed one half of the maximum bed displacement. For slower motions of the bed, the maximum wave amplitudes decreased. Similar features relating to the maximum amplitudes of waves propagating from the generation region in a three-dimensional fluid domain were obtained by Nakamura (1953), Kajiura (1963) and Momoi (1964). Momoi's numerical computations provide the most detailed description of the evolution of waves near the generation region for an instantaneous uplift of a circular section of the bed.

Using the integral solutions developed by the linear theory for specific bed deformations, several authors (see, for example, Keller 1963; Kajiura 1963) have examined the far-field wave behaviour using asymptotic methods such as the method of stationary phase to evaluate these integrals. These asymptotic results suggest that an oscillatory wave train develops in the far field and continues to disperse into its spectral components during propagation: hence, the amplitude of the leading wave decays and no waves of permanent form evolve. 
Since the general features of the waves obtained by these authors for both the near and far fields are based on a linear theory, their applicability is limited to bed deformations and the range of propagation for which nonlinear effects remain small. One of the primary objectives of this study is to determine the necessary conditions for the linear theory to remain valid in the generation region and to examine the effect of the growth of nonlinearities on wave behaviour during propagation.

Hwang \& Divoky (1970) have adopted a nonlinear theory and constructed a numerical model of tsunami generation and propagation which permits an arbitrary bed displacement and a variable water depth to be included in the model. Although this model does consider nonlinearities, the linear effects of frequency dispersion are omitted; hence, no insight into the possible importance of the interaction of nonlinear and linear effects in the far field is possible. Tuck \& Hwang (1972) have most recently considered waves generated by a bed displacement on a uniformly sloping beach using the linear long-wave equations to describe the motion. These equations omit both the linear effects of frequency dispersion and nonlinearities; hence, their applicability for describing tsunami propagation is limited.

Experimental studies of tsunami generation by bed displacements are rare. Takahasi \& Hatori (1962) and Takahasi (1963) reported a series of experiments in which waves were generated by an impulsive deformation of both circular and elliptical rubber membranes installed at the bottom of wave basins. Results from these experiments agreed qualitatively with the results predicted by previous linear theories although no detailed comparison was made. In the present study, experiments have been conducted using a unique laboratory facility which permits the theoretical model of tsunami generation and propagation to be accurately modelled in the laboratory.

\section{Theoretical analysis}

Consider a fluid domain $D$ as shown in figure 1 bounded above by the free surface $S_{f}$, bounded below by a solid boundary $S_{b}$ and unbounded in the direction of wave propagation, i.e. $-\infty<x<\infty$. Initially the fluid is at rest with the free surface and solid boundary defined by $y=0$ and $y=-h$, respectively. For $t>0$ the solid boundary is permitted to move in a prescribed manner given by $y=-h+\zeta(x ; t)$ such that $\lim _{|x| \rightarrow \infty} \zeta(x ; t)=0$. The resulting deformation of the free surface, which is to be determined, is given by $y=\eta(x ; t)$. If it is assumed that the fluid is incompressible and the flow irrotational a velocity potential $\phi=\phi(x, y ; t)$ such that the fluid velocity vector can be expressed as $\mathbf{q}=\nabla \phi$ is known to exist; hence, from the continuity equation,

$$
\nabla \cdot \mathbf{q}=\nabla^{2} \phi=0 \text { in } D .
$$

The kinematic boundary conditions to be satisfied on the free surface and the solid boundary are, respectively,

$$
\phi_{y}=\left\{\begin{array}{lll}
\eta_{t}+\phi_{x} \eta_{x} & \text { on } & y=\eta(x ; t) \\
\zeta_{t}+\phi_{x} \zeta_{x} & \text { on } & y=-h+\zeta(x ; t)
\end{array}\right.
$$




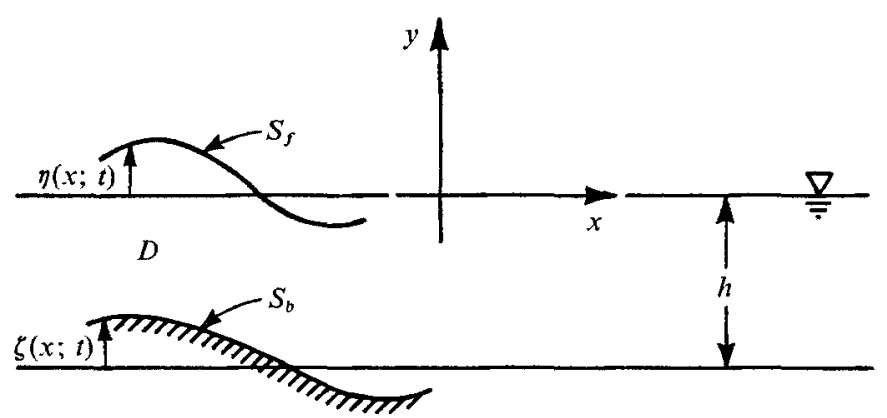

Frgure 1. Definition sketch of the fluid domain and co-ordinate system.

By further assuming the flow to be inviscid and surface energy effects to be negligible, the dynamic condition to be satisfied by fluid particles on the free surface may be written as

$$
\phi_{t}+\frac{1}{2}(\nabla \phi)^{2}+g \eta=0 \quad \text { on } \quad y=\eta(x ; t)
$$

The solution of (1)-(4) for a prescribed bed movement $\zeta(x ; t)$ is inherently difficult owing to the nonlinear terms in the boundary conditions and the unknown location of the free surface $a$ priori. The usual procedure for solving problems of this type is to circumvent these difficulties by substituting a linear approximation for the complete description of wave motion. In this approximation the nonlinear terms in the boundary conditions are omitted and the resulting equations are applied at the initial position of the boundaries. The linearized boundary conditions are given by

$$
\begin{aligned}
\phi_{y}(x, 0 ; t) & =\eta_{t}(x ; t), \\
\phi_{y}(x,-h ; t) & =\zeta_{t}(x ; t), \\
\phi_{t}(x, 0 ; t)+g \eta(x ; t) & =0 .
\end{aligned}
$$

Equations (5) and (7) are usually combined to yield the single free surface condition

$$
\phi_{t t}(x, 0 ; t)+g \phi_{y}(x, 0 ; t)=0 .
$$

A formal basis for using this linear approximation can be found by expanding the dependent variables as power series in terms of a small parameter $\epsilon$. Collecting: terms of the lowest order in $\epsilon$ yields the linear approximation; hence, the accuracy of this approximation is dependent on the magnitude of $\epsilon$. A physical interpretation of the parameter $\epsilon$ for the present problem and a more detailed discussion of nonlinear effects will be presented shortly.

Using the Laplace transform in $t$ and the Fourier transform in $x$, equations (1), (6), and (8) become

$$
\begin{gathered}
\bar{\phi}_{y y}(k, y ; s)-k^{2} \bar{\phi}(k, y ; s)=0 \\
\bar{\phi}_{y}(k,-h ; s)=s \bar{\zeta}(k ; s) \\
\bar{\phi}_{y}(k, 0 ; s)+\left(s^{2} / g\right) \bar{\phi}(k, 0 ; s)=0
\end{gathered}
$$


where the overbar on a function $f(x ; t)$ indicates that

$$
\bar{f}(k ; s)=\int_{-\infty}^{\infty} d x \int_{0}^{\infty} e^{i k x} e^{-s t} f(x ; t) d t .
$$

The transformed water surface elevation is found from (7) to be

Solution of (9) yields

$$
\bar{\eta}(k ; s)=-(s / g) \bar{\phi}(k, 0 ; s) .
$$

$$
\bar{\phi}(k, y ; s)=A(k ; s) \cosh k y+B(k ; s) \sinh k y .
$$

Substituting (14) into the boundary conditions (10) and (11) one obtains

$$
\bar{\phi}(k ; y ; s)=\frac{-g s \zeta(k ; s)}{\left(s^{2}+\omega^{2}\right) \cosh k h}\left[\cosh k y-\frac{s^{2}}{g k} \sinh k y\right],
$$

where $\omega^{2} \equiv g k \tanh k h$. Hence, the water surface elevation becomes

$$
\eta(k ; s)=s^{2} \bar{\zeta}(k ; s) /\left(s^{2}+\omega^{2}\right) \cosh k h .
$$

Inverting the Laplace and Fourier transforms yields

$$
\eta(x ; t)=\frac{1}{2 \pi} \int_{-\infty}^{\infty}\left\{\lim _{\Gamma \rightarrow \infty} \frac{1}{2 \pi i} \int_{\mu-i \Gamma}^{\mu+i \Gamma} \frac{s^{2} e^{-i k x} e^{s t} \bar{\zeta}(k ; s)}{\left(s^{2}+\omega^{2}\right) \cosh k h} d s\right\} d k,
$$

where the complex inversion integral for the Laplace transform has been used.

Before a further simplification of (17) can be made, specific bed deformations must be considered. Of special interest in the present study are two bed deformations whose spatial variation is taken to be a block section of the bed, symmetric about $x=0$, moving in the positive or negative vertical direction. The first displacement is given by

$$
\zeta_{e}(x ; t)=\zeta_{0}\left(1-e^{-\alpha t}\right) H\left(b^{2}-x^{2}\right) \quad(t \geqslant 0)
$$

where $H$ is the Heaviside step function. For this displacement (hereafter referred to as the exponential bed movement) the section of the bed in the interval $-b<x<b$ rises or falls to a maximum displacement $\zeta_{0}$ in an asymptotic manner (see figure 2). Note that a discontinuity exists in the bed velocity at $t=0$.

The second bed deformation of interest is given by

$$
\zeta_{s}(x ; t)=\zeta_{0}\left[\frac{1}{2}(1-\cos \pi t / T) H(T-t)+H(t-T)\right] H\left(b^{2}-x^{2}\right) \quad(t \geqslant 0) .
$$

For $0 \leqslant t<T$ the bed section in the interval $-b<x<b$ moves according to the function $\zeta_{0}\left[\frac{1}{2}(1-\cos \pi t / T)\right]$; hence the bed velocity is continuous. At $t=T$ the block section reaches the maximum displacement $\zeta_{0}$ and remains at this elevation for $t>T$ (see figure 2). This bed deformation will hereafter be referred to as the half-sine bed displacement.

Three parameters are necessary to characterize each of the bed displacements: a characteristic size $b$, an amplitude of movement $\zeta_{0}$ and a characteristic time $t_{c}$. For the exponential bed movement a characteristic time has been chosen such that 


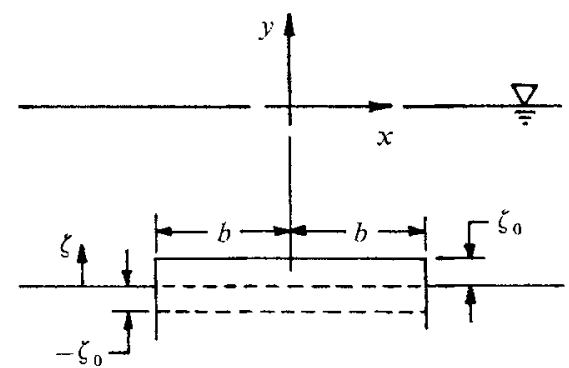

(a)

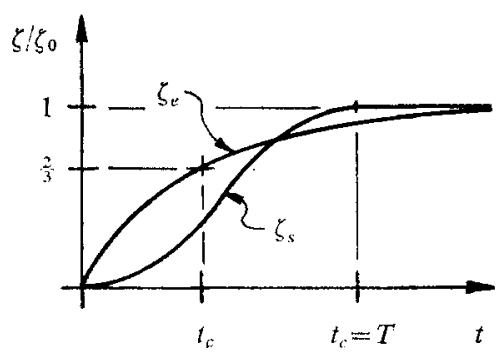

(b)

Figure 2. Bed deformation models: (a) spatial deformation; (b) time-displacement histories.

$t=t_{c}$ when $\zeta / \zeta_{0}=\frac{2}{3}$ (or $\left.t_{c}=1 \cdot 11 / \alpha\right)$. For the half-sine bed displacement the total time of movement $T$ will be defined as the characteristic time $t_{c}$. (These characteristic times are noted in figure 2.)

Laplace and Fourier transformation can now be applied to the bed motions described by (18) and (19) to yield

$$
\begin{gathered}
\bar{\zeta}_{e}(k ; s)=2 \zeta_{0} \frac{\sin k b}{k}\left[\frac{\alpha}{s(s+\alpha)}\right], \\
\bar{\zeta}_{s}(k ; s)=\zeta_{0} \frac{\sin k b}{k}\left\{\left(1+e^{\left.-s T^{\prime}\right)}\left[\frac{\kappa^{2}}{s\left(s^{2}+\kappa^{2}\right)}\right]\right\},\right.
\end{gathered}
$$

where $\kappa=\pi / T$. Substituting (20) into (17), performing the integration around the Bromwich contour, taking only the real part of the resulting integral and noting that the integrand is an even function of $k$, the water surface displacement resulting from an exponential bed movement is found to be

$$
\eta_{e}(x ; t)=-\frac{2 \zeta_{0}}{\pi} \int_{0}^{\infty} \frac{\cos k x \sin k b}{k \cosh k h}\left[\frac{\alpha^{2}}{\alpha^{2}+\omega^{2}}\right]\left[e^{-\alpha t}-\cos \omega t-\frac{\omega}{\alpha} \sin \omega t\right] d k .
$$

In a similar manner, the water surface displacement resulting from the halfsine bed movement is given by

$$
\begin{aligned}
\eta_{s}(x ; t)=\frac{\zeta_{0}}{\pi} \int_{0}^{\infty} \frac{\cos k x \sin k b}{k \cosh k h}\left\{\frac{\kappa^{2}}{\kappa^{2}-\omega^{2}}\right\}\{\cos \omega t-\cos \kappa t \\
+H(t-T)[\cos \omega(t-T)+\cos \kappa t]\} d k .
\end{aligned}
$$

The integration over $k$ in (22) and (23) cannot be performed in closed form; thus, numerical integration must be used.

\section{Aspects of nonlinearity: generation}

Since the solutions given by (22) and (23) are based on a linear approximation to the complete nonlinear description of wave behaviour, it is of major importance to determine the conditions necessary for these solutions to be applicable. For the problem under consideration, there are two regions of the fluid domain in 
which nonlinear effects may become significant. The first region is the neighbourhood of the deforming bed and will be referred to as the generation region. When the nonlinear effects are significant in this region, the linear description of wave behaviour becomes invalid for all time. (It should be emphasized that this statement is strictly valid only when the propagating wave energy is confined between parallel lines, i.e. the wave energy does not spread normal to the direction of wave propagation. When the wave energy does spread normal to the direction of wave propagation, a linear wave system may evolve from a nonlinear wave train.) The second region of the fluid domain where nonlinearities may become important is downstream of the generation region and arises as a result of the cumulative nature of nonlinear effects during wave propagation. A discussion of these nonlinear effects and the validity of the linear theory during wave propagation in the far field of this region will be presented in $\S 6$.

In order to determine the conditions necessary for the linear theory to be applicable in the generation region, the magnitude of the nonlinear terms which are omitted in this approximation (relative to the terms which are retained) must be estimated. Estimates of this type require that each dependent and independent variable be scaled in a manner such that each variable in its nondimensional form is approximately unity; hence, the magnitude of each term in the governing equations is indicated by its coefficient. During the time of the bed displacement, it would seem appropriate to scale the motion of a fluid particle near the bed deformation by quantities which are characteristic of the bed motion. In order to determine the magnitude of the nonlinear effects introduced during the generation process in this manner, it is advantageous to examine the non-integrated forms of the governing equations. Under the assumptions stated in the previous section these equations may be written as

$$
\begin{gathered}
u_{t^{*}}^{*}+u^{*} u_{x^{*}}^{*}+v^{*} u_{y^{*}}^{*}+\rho^{-1} P_{x^{*}}^{*}=0, \\
v_{t^{*}}^{*}+u^{*} v_{x^{*}}^{*}+v^{*} v_{y^{*}}^{*}+\rho^{-1} P_{y^{*}}^{*}+g=0, \\
u_{x^{*}}^{*}+v_{y^{*}}^{*}=0
\end{gathered}
$$

where $\rho$ and $P^{*}$ are the fluid density and pressure, respectively, and the asterisk superscript is used to indicate a dimensional variable. The boundary conditions are

$$
v^{*}= \begin{cases}\eta_{t^{*}}^{*}+u^{*} \eta_{x^{*}}^{*} & \text { on } y^{*}=\eta^{*}, \\ \zeta_{t^{*}}^{*}+u^{*} \zeta_{x^{*}}^{*} & \text { on } y^{*}=-h+\zeta^{*} .\end{cases}
$$

Assume for the moment that the bed displacement occurs so rapidly that the deformed water surface is similar in shape to the deformed bed at any time during the bed movement. (Bed movements of this type will be referred to as impulsive.) Then the resulting free-surface deformation has a characteristic length, amplitude and time which are equivalent to those of the deforming bed. The vertical motion of a water particle for this type of generation is dominated by the vertical motion of the bed; hence, the velocity $v^{*}$ should be scaled by a characteristic velocity of the moving bed, e.g. $\zeta_{0} / t_{c}$. For the specific bed deformations under consideration (where $\zeta_{x^{*}}^{*}=0$, except at $\left|x^{*}\right|=b$ ) the horizontal velocity $u^{*}$ results 
from wave motion only and is of order $\eta^{*}(g h)^{\frac{1}{2}} / h$; however, since the wave amplitude $\eta^{*}$ is of order $\zeta_{0}$, the appropriate velocity required to scale $u^{*}$ is $\zeta_{0}(g h)^{\frac{1}{2}} / h$. With the pressure $P^{*}$ scaled by $\rho g h$, the following scaling of variables appears to be appropriate for impulsive wave generation:

$$
\left.\begin{array}{l}
x^{*}=b x, \quad y^{*}=h y, \quad t^{*}=t_{c} t, \quad u^{*}=\left[\zeta_{0}(g h)^{\frac{1}{2}} / h\right] u, \\
v^{*}=\left(\zeta_{0} / t_{c}\right) v, \quad \eta^{*}=\zeta_{0} \eta, \quad P^{*}=\rho g h P, \quad \zeta^{*}=\zeta_{0} \zeta .
\end{array}\right\}
$$

Substitution of (29) into (24)-(28) yields

$$
\begin{gathered}
u_{t}+\left(\frac{t_{c}(g / h)^{\frac{1}{2}}}{b / h}\right)\left(\frac{\zeta_{0}}{h}\right) u u_{x}+\frac{\zeta_{0}}{h} v u_{y}+\left(\frac{t_{c}(g / h)^{\frac{1}{2}}}{b / h}\right)\left(\frac{\zeta_{0}}{h}\right)^{-1} P_{x}=0, \\
v_{t}+\left(\frac{t_{c}(g / h)^{\frac{1}{2}}}{b / h}\right)\left(\frac{\zeta_{0}}{h}\right) u v_{x}+\frac{\zeta_{0}}{h} v v_{y}+\left[t_{c}(g / h)^{\frac{1}{2}}\right]^{2}\left(\frac{\zeta_{0}}{h}\right)^{-1}\left[P_{y}+1\right]=0, \\
u_{x}+\left(\frac{t_{c}(g / h)^{\frac{1}{2}}}{b / h}\right)^{-1} v_{y}=0, \\
v=\left\{\begin{array}{cl}
\eta_{t}+\left(\frac{t_{c}(g / h)^{\frac{1}{2}}}{b / h}\right)\left(\frac{\zeta_{0}}{h}\right) u \eta_{x} & \text { on } y=\frac{\zeta_{0}}{h} \eta \\
\zeta_{t}+\left(\frac{t_{c}(g / h)^{\frac{1}{2}}}{b / h}\right)\left(\frac{\zeta_{0}}{h}\right) u \zeta_{x} & \text { on } y=-1+\frac{\zeta_{0}}{h} \zeta .
\end{array}\right.
\end{gathered}
$$

Examination of (30)-(34) indicates that the linear terms dominate wave behaviour if the following conditions are satisfied:

$$
\left(\frac{t_{c}(g / h)^{\frac{1}{2}}}{b / h}\right)\left(\frac{\zeta_{0}}{h}\right) \ll 1, \quad\left(\frac{\zeta_{0}}{h}\right) \ll 1,
$$

In addition, the boundary conditions on the free surface and solid boundary may be applied on $y=0$ and $y=-1$, respectively, as a first approximation when these conditions are satisfied. The two parameters in (35) consist of three dimensionless ratios that characterize the generation process: $\zeta_{0} / h$, which represents an amplitude scale; $b / h$, which represents a size scale; and $t_{c}(g / h)^{\frac{1}{2}}$, which represents a time scale. An interesting combination of two of these dimensionless numbers is given by the ratio of the time and size scales, which may also be written as $t_{c}(g h)^{\frac{1}{2}} / b$. The quantity $t_{c}(g h)^{\frac{1}{2}}$ is simply the distance a long gravity wave of small amplitude will travel in the limit $t_{c}$. Thus, if $t_{c}(g h)^{\frac{1}{2}} / b$ is much less than unity a major portion of the bed motion occurs before elevations (or depressions) of the water surface have an opportunity to leave the generation region. This results in an initial water surface deformation similar in shape to the deformed bed as was assumed in the scaling of variables given by (29). Thus, it appears from this analysis that for waves generated such that $t_{c}(g h)^{\frac{1}{2}} / b \ll 1$, i.e. impulsively, the primary restriction on the applicability of the linear theory during generation is that the disturbance amplitude scale $\zeta_{0} / h$ must remain much less than unity. (The parameter $t_{c}(g h)^{\frac{1}{2}} / b$ will hereafter be referred to as the time-size ratio.)

The magnitude of the nonlinear terms neglected in the linear approximation can also be determined during a bed deformation for which the bed motion occurs slowly (quantitatively this implies that the time-size ratio of the motion is much 
greater than unity). For displacements of this type (hereafter referred to as 'creeping') the water surface elevations (depressions) that occur have sufficient time to leave the source region during the bed motion. Near the end of the bed motion the displaced water is distributed over a length proportional to $t_{c}(g h)^{\frac{1}{2}}$, which would appear to be an appropriate length scale for the resulting wave. A characteristic amplitude for the displaced water can be found easily by equating the volume in the wave to the volume displaced by the deformed bed, which yields a characteristic amplitude of $\zeta_{0} b / t_{c}(g h)^{\frac{1}{2}}$. Time variations of this wave scale with the travel time of a long gravity wave across the source region; this time is proportional to $b /(g h)^{\frac{1}{2}}$. An appropriate horizontal velocity with which to scale $u^{*}$ is $\zeta_{0} b / t_{c} h$, which is found in a manner similar to that described previously for impulsive bed motions. Again assuming the vertical motion of a fluid particle in the source region to be dominated by the vertically moving bed, the following scaling of variables appears to be appropriate for a creeping bed displacement, i.e. $t_{c}(g h)^{\frac{1}{2}} / b \gg 1$ :

$$
\left.\begin{array}{l}
x^{*}=t_{c}(g h)^{\frac{1}{2}} x, \quad y^{*}=h y, \quad t^{*}=\left[b /(g h)^{\frac{1}{2}}\right] t, \quad u^{*}=\left(b \zeta_{0} / t_{c} h\right) u, \\
v^{*}=\left(\zeta_{0} / t_{c}\right) v, \quad \eta^{*}=\left[\zeta_{0} b / t_{c}(g h)^{\frac{1}{2}}\right] \eta, \quad \zeta^{*}=\left[\zeta_{0} b / t_{c}(g h)^{\frac{1}{2}}\right] \zeta, \quad P^{*}=\rho g h P .
\end{array}\right\}
$$

Rewriting (24)-(28) in terms of these non-dimensional variables yields

$$
\begin{gathered}
u_{t}+\left(\frac{t_{c}(g h)^{\frac{1}{2}}}{b}\right)^{-2}\left(\frac{\zeta_{0}}{h}\right) u u_{x}+\left(\frac{t_{c}(g h)^{\frac{1}{2}}}{b}\right)^{-1}\left(\frac{\zeta_{0}}{h}\right) v u_{y}+\left(\frac{\zeta_{0}}{h}\right)^{-1} P_{x}=0 \\
v_{t}+\left(\frac{t_{c}(g h)^{\frac{1}{2}}}{b}\right)^{-2}\left(\frac{\zeta_{0}}{h}\right) u v_{x}+\left(\frac{t_{c}(g h)^{\frac{1}{2}}}{b}\right)^{-1}\left(\frac{\zeta_{0}}{h}\right) v v_{y}+\left[t_{c}(g / h)^{\frac{1}{2}}\right]\left(\frac{b}{h}\right)\left(\frac{\zeta_{0}}{h}\right)^{-1}\left(P_{y}+1\right)=0 \\
u_{x}+\frac{t_{c}(g h)^{\frac{1}{2}}}{b} v_{y}=0 \\
v=\left\{\begin{array}{lll}
\eta_{t}+\left(\frac{t_{c}(g h)^{\frac{1}{2}}}{b}\right)^{-2} u \eta_{x} & \text { on } \quad y=\left(\frac{t_{c}(g h)^{\frac{1}{2}}}{b}\right)^{-1}\left(\frac{\zeta_{0}}{h}\right) \eta \\
\zeta_{t}+\left(\frac{t_{c}(g h)^{\frac{1}{2}}}{b}\right)^{-2} u \zeta_{x} & \text { on } \quad y=-1+\left(\frac{t_{c}(g h)^{\frac{1}{2}}}{b}\right)^{-1}\left(\frac{\zeta_{0}}{h}\right) \zeta
\end{array}\right.
\end{gathered}
$$

Examination of (37)-(41) indicates that the linear theory should provide an accurate approximation of the wave behaviour if the following conditions are satisfied:

$$
\left(\frac{t_{c}(g h)^{\frac{1}{2}}}{b}\right)^{-2}\left(\frac{\zeta_{0}}{h}\right) \ll 1, \quad\left(\frac{t_{o}(g h)^{\frac{1}{2}}}{b}\right)^{-1}\left(\frac{\zeta_{0}}{h}\right) \ll 1 .
$$

The original assumption in this development was $t_{c}(g h)^{\frac{1}{2}} b \gg 1$; hence, the conditions indicated by (42) are always satisfied for creeping bed motions regardless of the magnitude of the disturbance amplitude scale (which has an effective upper bound of unity). This analysis also suggests that the linearized boundary conditions $v=\eta_{t}$ and $v=\zeta_{t}$ may be applied on $y=0$ and $y=-1$, respectively, during a creeping bed motion even when the total bed displacement is equal to the water depth. 


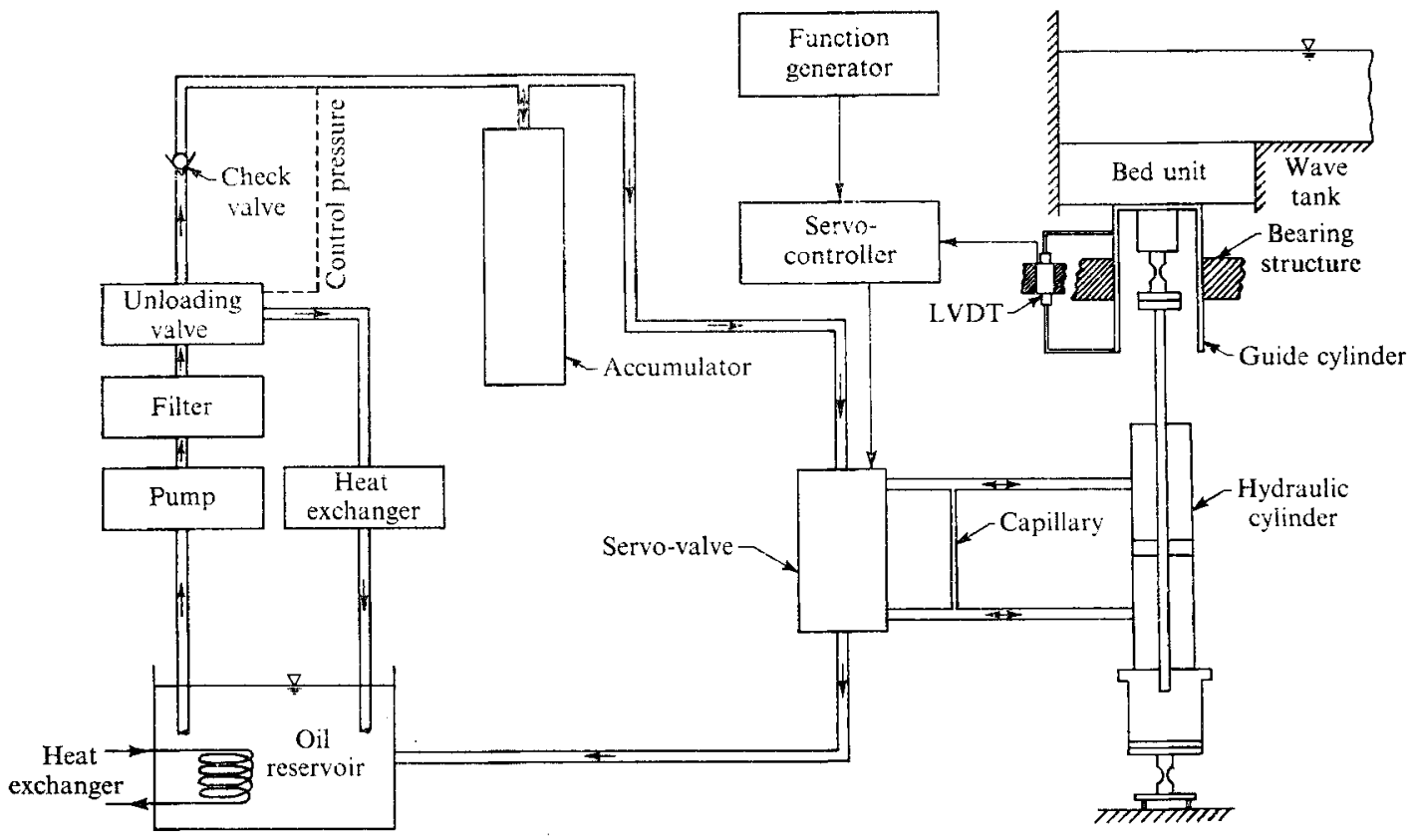

FIGURE 3. Schematic drawing of the bottom wave generator.

\section{Experimental apparatus}

A series of experiments was conducted in the laboratory in a wave tank $103.8 \mathrm{ft}$ long, $2 \mathrm{ft}$ deep and $15 \frac{1}{2} \mathrm{in}$. wide. The vertical side walls of the tank were constructed of glass throughout. The bottom wave generator was located at the upstream end of the wave tank and a wave energy dissipation system was located at the downstream end. Owing to the symmetry about $x=0$ of the simple bed deformations to be modelled, the origin of the theoretical model was represented in the laboratory by the vertical wall at the upsteam end of the wave tank; hence, only half of the bed deformation and fluid domain was modelled experimentally.

In order to model the bed deformations given by (18) and (19) in the laboratory, a bottom wave generator was required in which both the time-displacement history of a block section of the bed and the characteristic parameters of the bed deformations, i.e. $\zeta_{0}, b$ and $t_{c}$, could easily be controlled and varied. A hydraulic servo-system was developed to meet these requirements. A schematic drawing of the wave generator system is shown in figure 3 . The function generator provides a d.c. voltage which varies with time in a manner that is proportional to the desired time-varying displacement of the bed section. The servo-system converts the time-dependent electrical signal into a time-dependent displacement of the bed unit proportional to it. The hydraulic supply unit provides the pressure necessary to displace the moveable bed section. (A rubber seal is attached to the bottom of the bed unit around its periphery so that the fluid domain is confined to the region above the unit.) A photograph of the bottom wave generator installed in the wave tank is shown in figure 4 (plate 1). 
The wave generator and the end of the wave tank were constructed in such a manner that bed units with different lengths $b$ could be used; two bed units with lengths of $30.5 \mathrm{~cm}$ and $61 \mathrm{~cm}$ were used in the experiments (the larger bed unit is shown in figure 4). Five water depths ranging from 5 to $50 \mathrm{~cm}$ were used and provided a range of disturbance size scales given by $0 \cdot 61 \leqslant b / h \leqslant 12 \cdot 2$. The maximum total bed displacement $\zeta_{0}$ used for rapid bed movements was $\pm 5 \mathrm{~cm}$; hence, a disturbance amplitude scale of $\left|\zeta_{0} / h\right|=1$ was possible for the largest bed, i.e. $b / h=12 \cdot 2$. The smallest characteristic times for which the wave generator was able to follow the programmed bed displacement accurately with $\zeta_{0} \leqslant 5 \mathrm{~cm}$ were $t_{c}=0.073 \mathrm{~s}$ for the exponential bed displacement and $t_{c}=0.043 \mathrm{~s}$ for the half-sine bed displacement. Both the programmed and actual timedisplacement histories of the bed unit could be recorded simultaneously during an experiment; the characteristic time $t_{c}$ used in the reduction of data was always determined from the actual bed motion. (It should be noted that the discontinuity in the bed velocity at $t=0$ for the exponential bed motion is necessarily smoothed by the mechanical system and the range of impulsive motions for this time-displacement history was primarily limited by this smoothing process. A more detailed discussion of the wave generator and its response characteristics has been presented by Hammack (1972).)

Wave amplitudes at various positions in the fluid domain were measured electronically using parallel-wire resistance gauges and an oscillograph recorder. In most experiments the change in water surface elevation was measured at $x=0$ and $x=b$ and at three additional locations downstream of the source region; the downstream positions varied according to the water depth. Each wave gauge was calibrated before an experiment, which was completed within minutes of calibration.

\section{Presentation of results in the generation region}

The presentation of experimental and theoretical results for the source region of the fluid domain has two primary objectives: (i) to determine whether the linear and nonlinear behaviour suggested by the analysis in $\S 3$ for wave generation exists and (ii) to provide a simple method for approximating certain gross features of the primary waves which might occur in the source region of actual tsunamigenic earthquakes. Extrapolation of the results from this simple model study of tsunami generation to prototype phenomena is necessarily limited; hence, emphasis is primarily given to the examination of the maximum amplitude and relevant periods of the main wave which is generated rather than the detailed structure of the entire wave system. Results are presented for two locations in the generation region: $x / h=0$, which corresponds to the centre-line of the bed deformation (or to the position of the upstream end wall in the wave tank), and $x / h=b / h$, which is the downstream edge of the bed deformation. A knowledge of wave behaviour at the latter position is especially important since measurements here indicate the type of wave system propagating from the source region. 


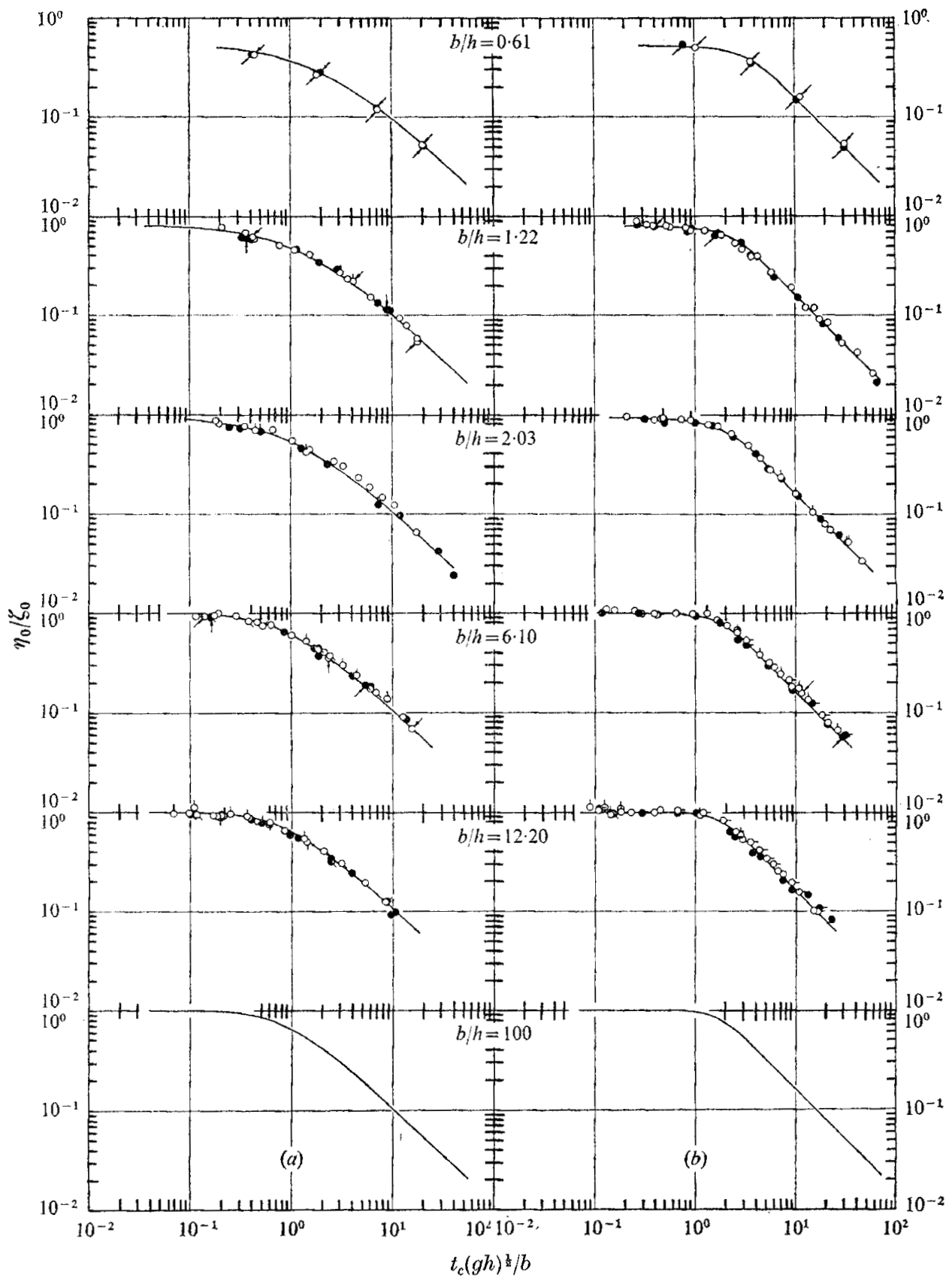

FigURE 5. Variation of the relative wave amplitude $\eta_{0} / \zeta_{0}$ with the time-size ratio $t_{c}(g h)^{\frac{1}{2}} / b$ at $x / h=0$. (a) Exponential bed motion. (b) Half-sine bed motion. - - linear theory; $\mathrm{O}, \zeta_{0}>0 ; \mathbf{O}, \zeta_{0}<0$. Arrows indicate data for which $b=30 \cdot 5 \mathrm{~cm}$; for all other data $b=61$ cm.

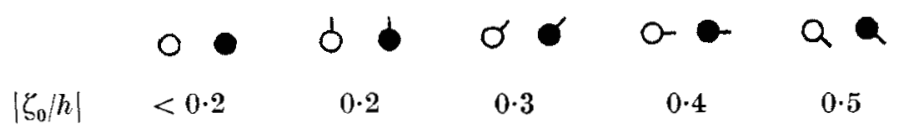




\subsection{Maximum wave amplitudes}

Experimental and theoretical results for the variation of the ratio of the maximum wave amplitude $\eta_{0}$ to the total bed displacement $\zeta_{0}$ as a function of the timesize ratio $t_{c}(g h)^{\frac{1}{2}} / b$ at $x / h=0$ are shown in figures $5(a)$ and $(b)$. Data are presented separately for each of the five size scales investigated experimentally and for both the exponential and half-sine bed motions; theoretical results alone are presented for the largest size scale $(b / h=100)$.

Examination of the results in figures $5(a)$ and $(b)$ indicates that the linear theory accurately predicts the relative wave amplitude $\eta_{0} / \zeta_{0}$ for both positive and negative bed motions over the full range of generation parameters $\left(\zeta_{0} / h\right.$, $b / h$ and $\left.t_{c}(g h)^{\frac{1}{2}} / b\right)$ investigated. No significant nonlinear effects are apparent even for data where the total bed displacement is equal to half the fluid depth and the motion occurs rapidly, i.e. $t_{c}(g h)^{\frac{1}{2}} / b \ll 1$. (The apparent absence of significant nonlinear effects at $x / h=0$ for large disturbance amplitude scales and small time-size ratios does not conform to the behaviour suggested by the analysis in §3.) The variation of the relative wave amplitude with the time-size ratio is similar for each of the size scales investigated. For rapid bed motions such that $t_{c}(g h)^{\frac{1}{2}} / b \ll 1$ (recall that motions of this type were termed impulsive in $\S 3$ ), the relative wave amplitude becomes constant and equal to unity for the three larger size scales. The maximum value of the relative wave amplitude for the smaller size scales is less than unity and decreases with size scale; this behaviour is a result of the elliptic $\left(\nabla^{2} \phi=0\right)$ nature of the response of the fluid field to an impulsive boundary condition (see Hammack (1972) for a detailed discussion of this behaviour). As the time-size ratio becomes very large the relative wave amplitude becomes inversely proportional to it in a manner similar to the behaviour suggested by the scaling of wave amplitudes for creeping bed motions $\left(t_{c}(g h)^{\frac{1}{2}} / b \gg 1\right)$ discussed in $\$ 3$. Comparison of the theoretical results for $b / h=12 \cdot 2$ and $b / h=100$ in figures $5(a)$ and $(b)$ for the exponential and half-sine bed motions, respectively, indicates that the relative wave amplitude does not vary appreciably between these two size scales.

Experimental and theoretical results for the variation of the relative wave amplitude with the time-size ratio at the leading edge of the bed deformation $(x / h=b / h)$ are shown in figures $6(a)$ and $(b)$. Results for both the exponential and half-sine bed displacements are presented in the same manner as the results shown in figure 5. The theoretical variation for each size scale again indicates that the relative wave amplitude reaches a maximum and remains constant for impulsive bed motions; for the larger size scales the maximum wave amplitude becomes equal to half the total bed displacement. For the creeping range of the time-size ratios $\left(t_{c}(g h)^{\frac{1}{2}} / b \gg 1\right)$ the relative wave amplitude again becomes inversely proportional to the time-size ratio. The agreement of the linear theory with the experimental results is no longer as complete as for $x / h=0$ over the full range of generation parameters investigated. Significant nonlinear effects are now apparent in the impulsive $\left(t_{c}(g h)^{\frac{1}{2}} / b \ll 1\right)$ and transitional $\left(t_{c}(g h)^{\frac{1}{2}} / b \approx 1\right)$ range of time-size ratios when $\left|\zeta_{0} / h\right|>0 \cdot 2$. In the creeping range of time-size ratios, nonlinear effects are not apparent even when the bed displacement is 


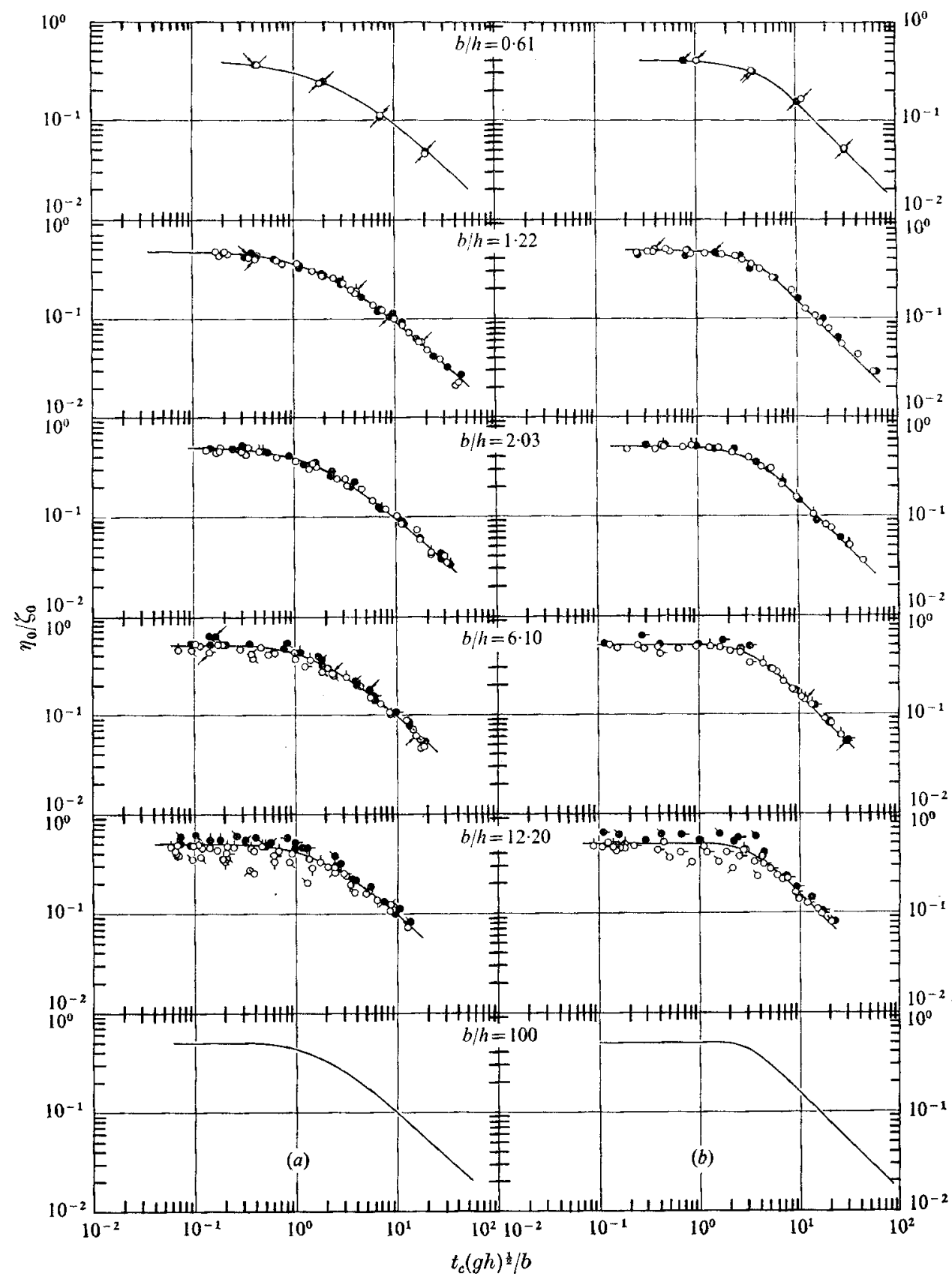

FIGURE 6. Variation of the relative wave amplitude $\eta_{0} / \zeta_{0}$ with the time-size ratio $t_{c}(g h)^{\frac{1}{1}} / b$ at $x / h=b / h$. (a) Exponential bed motion. (b) Half-sine bed motion. - - linear theory; $0, \zeta_{0}>0 ;, \zeta_{0}<0$. Arrows indicate data for which $b=30.5 \mathrm{~cm}$; for all other data $b=61 \mathrm{~cm}$.

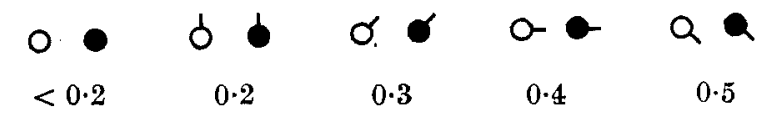
$\left|\zeta_{0} / h\right|<0.2$
Q
$-0$ 
equal to the water depth. These nonlinear effects for impulsive bed motions, which are associated with the disturbance amplitude scale, and the absence of similar nonlinearities for creeping motions of the bed conforms to the wave behaviour suggested by the analysis in in $\$ 3$. Comparison of the theoretical results for $b / h=12 \cdot 2$ and $b / h=100$ in figures $6(a)$ and $(b)$ indicates that the relative wave amplitude at $x / h=b / h$ does not vary appreciably between these larger size scales.

\subsection{Wave profiles in the generation region}

In addition to the maximum amplitude of the leading wave, it is also of interest to observe the complete temporal variation of the waves in the generation region. Experimental and theoretical wave signatures for both the exponential and halfsine bed displacements at $x / h=0$ and $x / h=b / h$ are shown in figures $7(a)$ and (b); typical wave profiles resulting from impulsive, transitional and creeping motions of the bed are illustrated. Note that the generation parameters for each bed motion have been chosen such that the linear theory should be applicable for predicting the maximum wave amplitude at each position in the source region.

Comparison of the theoretical computations with the measured profiles for impulsive bed motions in figures $7(a)$ and $(b)$ indicates that the linear theory does provide an accurate description of wave behaviour in the source region during the initial period of wave evolution: however, discrepancies between theory and experiment occur in the trailing region of the leading wave. The linear theory appears to agree very well with experimental measurements for transitional or creeping bed motions over the complete interval of wave evolution shown.

For impulsive bed motion, both the experimental and theoretical results indicate that the water surface initially moves to a maximum displacement of $\zeta_{0}$ and $\frac{1}{2} \zeta_{0}$ at $x / h=0$ and $x / h=b / h$, respectively. After reaching a maximum displacement, the water level remains stationary for an interval of time and then rapidly returns to the still-water level, about which it oscillates in a damped manner. The main portion of the wave energy is concentrated in the leading wave, whose shape resembles that of the deformed bed. Wave profiles for both the exponential and half-sine bed displacement are similar; hence, the final shape of the deformed bed and not the time-displacement history of its movement appears to be the primary factor in determining the wave signatures in the source region for these impulsive bed motions.

For transitional bed motions, the water surface initially moves to a maximum displacement and immediately begins returning to the still-water level. The wave signatures at corresponding positions in the generation region are no longer similar for the exponential and half-sine bed displacements; hence, the time-displacement history of these bed motions becomes more important in determining the wave signature.

The wave signatures for the creeping bed motions clearly show the influence of the time-displacement history of the bed movement. For the exponential bed displacement the wave profiles at both positions resemble a bore; the water level rises to a maximum elevation and then begins to return to the still-water level 


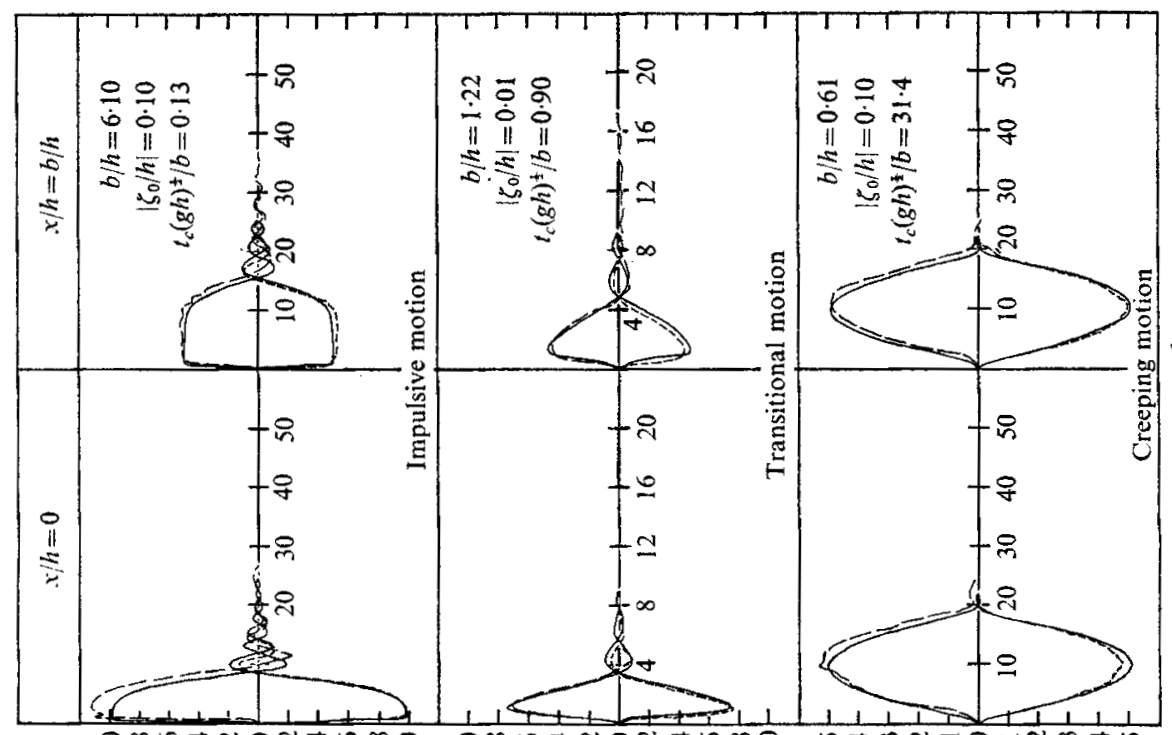

.

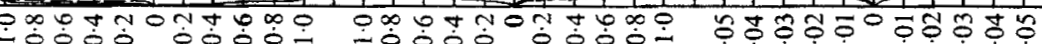
$=120^{\circ}$

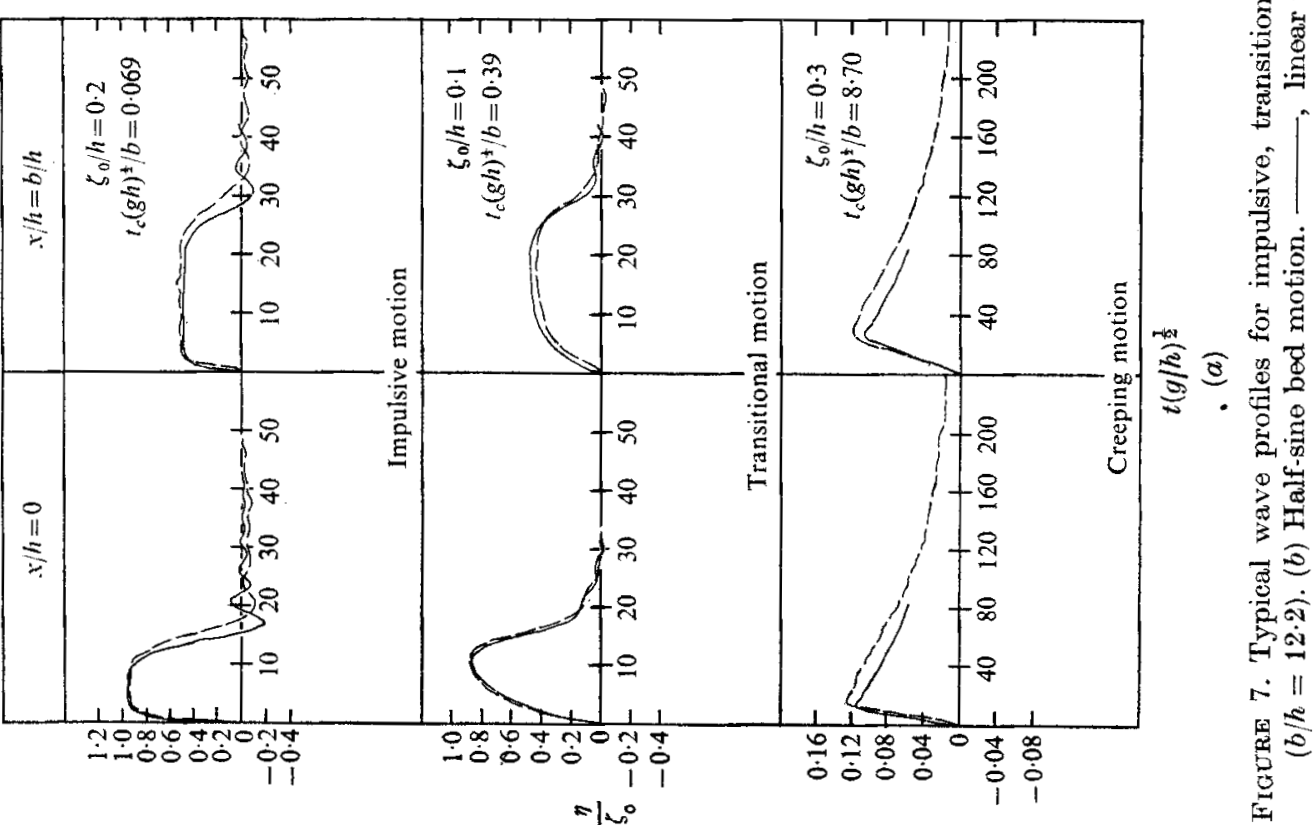


at a very slow rate. For the half-sine bed motion the water rises (or falls) to a maximum (or minimum) elevation and then returns to the still-water level, forming a symmetric wave. (In fact, the wave profiles for the creeping half-sine bed motion resemble the time-velocity history of the bed motion.) These results clearly demonstrate the importance of the time-displacement history of the bed motion in determining the resulting wave signatures for creeping movements.

For the complex wave profiles shown in figures $7(a)$ and $(b)$ no single period exists which adequately describes the leading wave. In order to describe the general features of the leading waves resulting from these bed motions, three periods are required: (i) the time $t_{r}$ at which the water surface reaches its maximum displacement, (ii) the time $t_{f}$ at which the water level begins to return towards the still-water level, and (iii) the time $t_{n}$ at which the water surface again reaches itsinitial position (all times are measured from $t=0$ ). It is observed from figures $7(a)$ and $(b)$ that $t_{r}=t_{f}$ for all waves generated by a transitional or creeping motion of the bed and that $t_{n}$ becomes exceedingly large for creeping bed motions with an exponential time-displacement history. A knowledge of these three periods as well as the maximum wave amplitude $\eta_{0}$ provides a general description of the overall structure of the leading waves shown in figures $7(a)$ and $(b)$. (The periods $t_{r}, t_{f}$ and $t_{n}$ will hereafter be referred to as the rise time, fall time and nodal time respectively.)

\subsection{Leading wave periods at $x / h=b / h$}

Experimental and theoretical results for the periods $t_{r}, t_{f}$ and $t_{n}$ of the leading wave at $x / h=b / h$ are shown in figure 8 for a bed motion with an exponential time-displacement history. The wave periods have been normalized by the characteristic time $t_{c}$ of the bed motion and are shown separately as a function of the time-size ratio for each disturbance size scale. Because of the difficulty encountered in defining $t_{n}$ for creeping bed motions, no data or theory is shown in figure 8 for this range of time-size ratios.

A comparison of theory with experiments in figure 8 indicates that nonlinear effects appear to be significant in determining the time ratios $t_{r} / t_{c}$ and $t_{n} / t_{c}$ for impulsive or transitional bed motions when $\zeta_{0} / h>0 \cdot 2$. (Similar nonlinear effects do not appear to be present for large negative displacements of the bed.) It is also observed in figure 8 that for creeping bed motions (where $t_{r}=t_{f}$ and $t_{n}$ is undefined) the time ratio $t_{r} / t_{c}$ is inversely proportional to the time-size ratio and no nonlinear effects appear to be present regardless of the magnitude of the disturbance amplitude scale. Comparison of the theoretical computations for $b / h=12 \cdot 2$ and $b / h=100$ indicates that the rise-time ratio $t_{r} / t_{c}$ and the nodal-time ratio $t_{n} / t_{c}$ do not vary appreciably for this increase in size scales. The fall-time ratio $t_{f} / t_{c}$ for impulsive bed motions appears to be changing in a manner such that the ratio $t_{n} / t_{f}$ of the nodal and fall times is approaching unity; hence, the rear portion of the wave is becoming steeper.

The time ratios for the leading waves generated by a half-sine bed displacement are shown in figure 9. The rise-time ratio for each size scale is asymptotic to a value of unity for impulsive bed motions and to a value of one half for creeping 


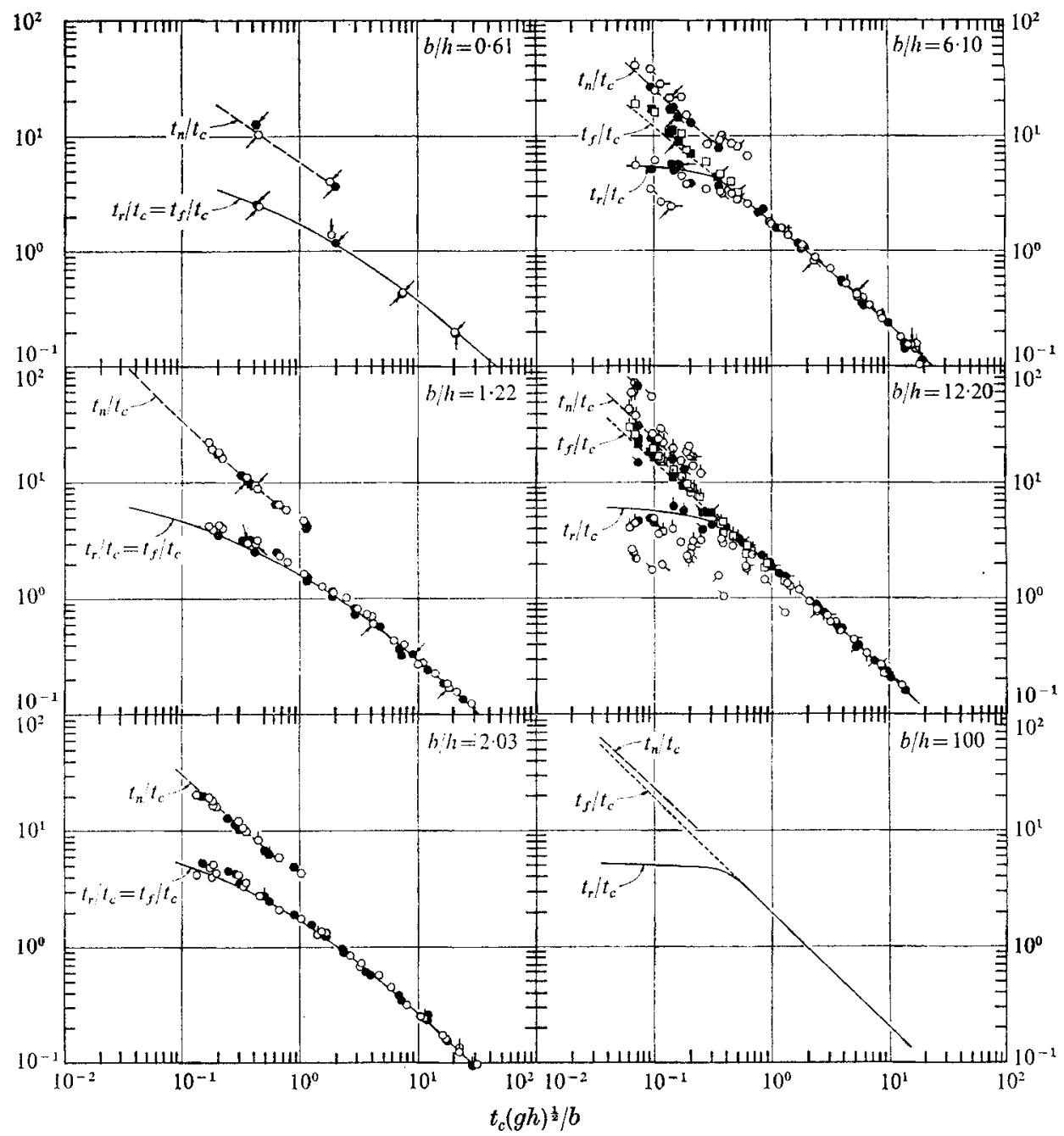

Frgure 8. Variation of the time ratios for the leading wave with the time-size ratio $t_{c}(g h)^{\frac{1}{2}} / b$ at $x / h=b / h$ for an exponential bed motion. Arrows indicate data for which $b=30.5 \mathrm{~cm}$; for all other data $b=61 \mathrm{~cm}$.

Linear theory $\quad$ Measured, $\zeta_{0}>0$

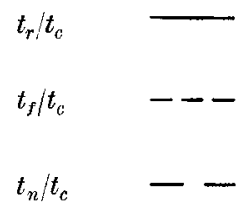

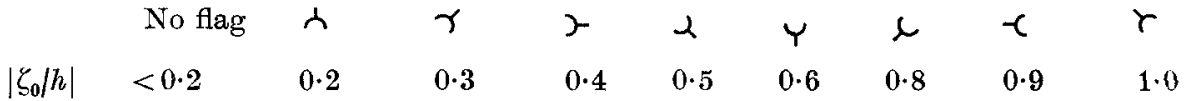


bed movement. The nodal-time ratio also approaches unity in the creeping range of time-size ratios; hence, since $t_{r}=t_{f}$ for these motions, a symmetric wave as shown in figure 7 is generated. Strong nonlinear effects based on the magnitude of the disturbance amplitude scale are not apparent in figure 9 for either the riseor fall-time ratios; however, the scatter of the rise-time measurements about the computed variation may have masked any weak nonlinear effects for this period. The nodal-time ratio does exhibit strong nonlinear effects as the disturbance amplitude scale becomes large. In a manner similar to that observed in figure 8 for the exponential bed displacement, the computed variations of the rise- and nodal-time ratios for $b / h=12 \cdot 2$ and 100 do not vary appreciably. The fall-time ratio again changes in a manner which indicates that the trailing portion of the lead wave generated by an impulsive motion is becoming steeper as the size scale increases.

\subsection{Conclusions and a comment on application of results to prototype phenomena}

The results presented in the previous sections have established the importance of the generation parameters $\left(\zeta_{0} / h, b / h\right.$ and $\left.t_{c}(g h)^{\frac{1}{2}} / b\right)$ in determining the wave structure in the source region. For impulsive bed motions $\left(t_{c}(g h)^{\frac{1}{2}} / b \ll 1\right)$ the initial wave structure resembles the final shape of the deformed bed; the exact time-displacement history of the motion appears to have only minor effects on the resulting wave behaviour. The wave structure for creeping bed motions $\left(t_{c}(g h)^{\frac{1}{2}} / b \gg 1\right)$ is strongly dependent on the time-displacement history of the movement; in fact, the resulting wave profiles resemble the time-velocity history of the bed motion. Nonlinear effects are observed to become significant at $x / h=b / h$ for impulsive bed motions or transitional bed motions $\left(t_{c}(g h)^{\frac{1}{2}} / b \approx 1\right)$ when the total bed displacement exceeds approximately $20 \%$ of the water depth, i.e. for $\left|\zeta_{0} / h\right|>0 \cdot 2$. Nonlinear effects are not observed at $x / h=0$ or for creeping bed motions at $x / h=b / h$ regardless of the magnitude of the disturbance amplitude scale. In order to extrapolate any of the conclusions based on this study to prototype phenomena, typical magnitudes of these generation parameters for tsunamigenic earthquakes are required. Although numerous seaquakes have occurred which have generated tsuanmis, very little information is available regarding the tectonic deformations of the sea bed which occur during these earthquakes. The best-documented tsunamigenic earthquake for which information of this type exists is the Alaskan earthquake of 27 March 1964; details of the tectonics for this earthquake have been presented by Plafker (1969). A tsunami was generated during this earthquake by the vertical uplift of the sea bed in an elongated area encompassing the continental shelf near the Gulf of Alaska. Examination of typical cross-sections of the sea-bed uplift in this elongated source region (presented by Plafker) indicate that a maximum vertical uplift of $30 \mathrm{ft}$ occurred in a small region where the water depth was approximately $300 \mathrm{ft}$; hence, since the uplift is smaller and the water depth generally larger elsewhere, the disturbance amplitude scale is bounded by $\zeta_{0} / h<0.1$. The half-width of the uplift region is observed from Plafker's results to be approxi- 


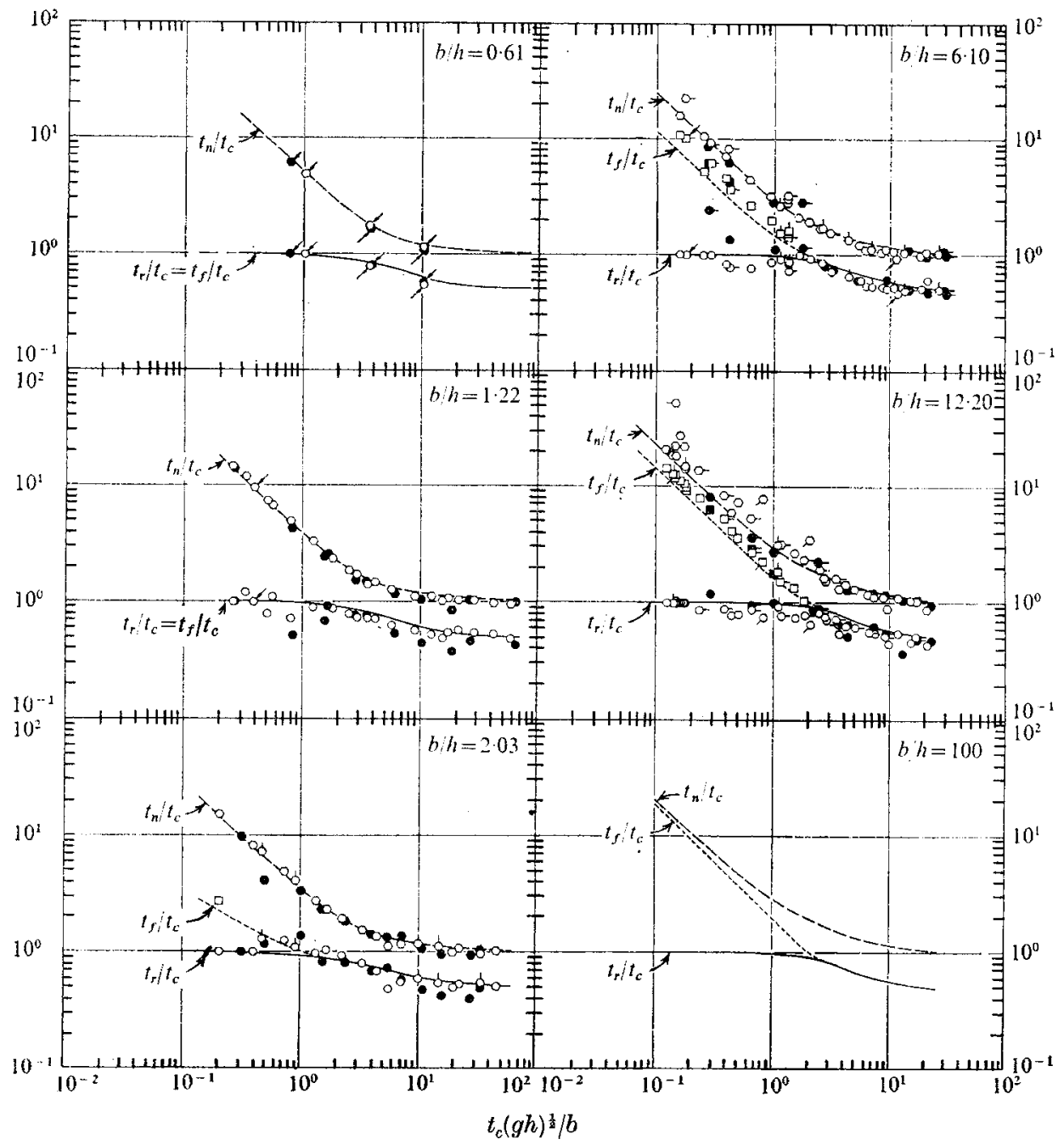

FIGURE 9. Variation of the time ratios for the leading wave with the time-size ratio $t_{c}(g h)^{\frac{1}{2}} / b$ at $x / h=b / h$ for a half-sine bed motion. Arrows indicate data for which $b=30.5 \mathrm{~cm}$; for all other $\operatorname{data} b=61 \mathrm{~cm}$.

Linear theory

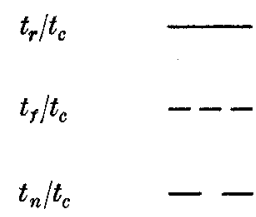

Measured, $\zeta_{0}>0$

0

0
Measured, $\zeta_{0}<0$

\begin{tabular}{|c|c|c|c|c|c|c|c|c|c|}
\hline & No flag & $\alpha$ & $\gamma$ & 2 & $\alpha$ & $Y$ & 2 & $-c$ & $r$ \\
\hline$\left|\zeta_{0} / h\right|$ & $<0.2$ & $0 \cdot 2$ & $0 \cdot 3$ & 0.4 & $0 \cdot 5$ & $0 \cdot 6$ & 0.8 & 0.9 & \\
\hline
\end{tabular}


mately 50 miles while the average water depth in this region is approximately $600 \mathrm{ft}$; hence, an approximate disturbance size scale is given by $b / h \approx 450$. No instrument records exist which indicate the time-displacement history of the ground motion during this earthquake; however, on the basis of measurements of an atmospheric gravity wave also generated by the Alaskan earthquake, Van Dorn (1964) suggests that the ground motion must have occurred in 2 to $6 \mathrm{~min}$. Using characteristic times $t_{c}$ of 2 and $6 \mathrm{~min}, b=50$ miles and $h=600 \mathrm{ft}$, the time-size ratio is found to lie in the range $0.06<t_{c}(g h)^{\frac{1}{2}} / b<0 \cdot 18$.

If the approximate values of the generation parameters presented above are indeed typical of tsunamigenic earthquakes, the following conclusions may be stated: (i) the bed motions are impulsive; hence, the permanent spatial deformation of the sea bed and not the time-displacement history of its motion is of primary importance in determining wave structure near the source region; (ii) nonlinear effects are not significant in defining initial wave behaviour; and (iii) the maximum amplitude of the wave system propagating from the source region is equal to half the maximum bed displacement. If the spatial deformation of the sea bed consists primarily of uniform uplift or downthrow, the leading wave characteristics $\left(\eta_{0}, t_{r}, t_{f}\right.$ and $\left.t_{n}\right)$ can be approximated from the results presented in figures 6 and 8 or figures 6 and 9 once estimates of $\zeta_{0}, b, h$ and $t_{c}$ are known. The results presented in these figures for $b / h=100$ can be used for larger size scales since these characteristics of the leading wave were found to be insensitive to increases in the size scale for $b / h>12 \cdot 2$. (See Hammack (1972) for a discussion of the use of these results for predicting the leading wave structure of the Alaskan tsunami.)

\section{Aspects of nonlinearity: propagation}

Once a wave has been generated and propagates into the fluid domain downstream of the generation region (where the solid boundary remains stationary with time and the water depth is uniform), the magnitude of nonlinear effects is determined by properties of the propagating wave. It is well known for long waves that the magnitude of the nonlinear terms in the governing equations is indicated by the ratio of the maximum wave amplitude, $\eta_{0}$, to the water depth $h$, i.e. by $\eta_{0} / h$. The magnitude of the linear terms is proportional to the square of the ratio of the water depth to a characteristic length $l$ of the wave in its direction of propagation, i.e. $(h / l)^{2}$. Hence, the relative importance of nonlinear and linear effects in a long wave propagating in a two-dimensional fluid domain is indicated by the ratio

$$
\frac{\text { nonlinear effects }}{\text { linear effects }} \propto \frac{\eta_{0} / h}{(h / l)^{2}}=\frac{\eta_{0} l^{2}}{h^{3}}=U .
$$

For $U \ll 1$ the linear effects of frequency dispersion dominate changes in the wave structure; hence a linear theory is applicable. For $U \gg 1$ the nonlinear effects (or amplitude dispersion) control the wave behaviour and a purely nonlinear description of wave motion is applicable. When $U$ is about unity, amplitude and frequency dispersion play an equal role in determining wave behaviour 
and both effects must be retained in the description of motion. (The ratio given by (43) will be referred to herein, as is common in much of the current literature, as the Ursell number based on the discussion of this parameter presented by Ursell (1953); however, earlier authors such as Stokes (1847) and especially Korteweg \& de Vries (1895) apparently understood the significance of this ratio in describing wave motion.)

When a wave system is initially described by a linear theory, the length of time during propagation for which the linear theory remains applicable is governed by the behaviour of $U$ during propagation. In order to examine this behaviour for a particular case which is relevant to the waves generated by the bed deformations presented in $\$ 2$, consider an initial condition where

$$
\eta(|x|<b ; 0)=\text { constant }=\zeta_{0}, \quad \eta(|x|>b ; 0)=0
$$

and the bed is defined by $y=-h$. (The results in the previous section indicate that a water surface deformation similar to this would exist at the end of an impulsive block upthrust of the bed for large disturbance size scales.) Using the solution for the large-time wave behaviour resulting from this initial condition presented by Jeffreys \& Jeffreys (1946, p. 485), Ursell (1953) demonstrated that near the wave front $U$ behaves as follows (with notation appropriate to this problem):

$$
U \sim\left(\frac{\zeta_{0}}{h}\right)\left(\frac{b}{h}\right)\left[t(g / h)^{\frac{1}{2}}\right]^{\frac{1}{3}}
$$

From (44) it is observed that $U$ grows with time like $t^{\frac{1}{2}}$; hence, regardless of how small nonlinear effects are initially, eventually they will become important. Note that (44) also indicates the importance of the disturbance amplitude and disturbance size scales in determining the length of time for which the linear theory is applicable; the time of applicability behaves like $\left(\zeta_{0} / h\right)^{-3}(b / h)^{-3}$. Although the above analysis has been applied to waves resulting from a particular initial condition, Meyer (1967) has shown that the linear theory also eventually becomes inapplicable for long waves of transition in a two-dimensional fluid domain.

As noted previously, when a wave train evolves into a state such that $U$ is about unity, the description of wave motion must include both frequency and amplitude dispersion. Approximate equations governing wave motions of this type have been presented by several authors including Korteweg \& de Vries (1895). The approximate equation found by these authors describing the propagation of a long wave in the direction of increasing $x$ is

$$
\eta_{t}+\left(1+\frac{3}{2} \eta\right) \eta_{x}+\frac{1}{6} \eta_{x x x}=0
$$

where $\eta=\eta^{*} / h, x=x^{*} / h$ and $t=t^{*}(g / h)^{\frac{1}{2}}$. (Equation (45) will hereafter be referred to as the $\mathrm{KdV}$ equation.) Another equation presented by Peregrine (1966) which describes wave motion to the same order of approximation as the $\mathrm{KdV}$ equation is given by

$$
u_{t}+\left(1+\frac{3}{2} u\right) u_{x}-\frac{1}{6} u_{x x t}=0,
$$


where $u=u^{*} /(g h)^{\frac{1}{2}}$ is the mean horizontal velocity of the fluid. The water surface elevation $\eta$ is related to the velocity $u$ to the same order of approximation by

$$
\eta=u+\frac{1}{4} u^{2}-\frac{1}{6} u_{x x}
$$

Even though (45) and (46) have the same formal justification as a description of wave motion, Benjamin, Bona \& Mahony (1972) have recently advocated (46) as a preferable model equation for wave propagation owing to significant differences in basic mathematical and computational properties of the two equations. (Equation (46) has been adopted for use in this study and for convenience will hereafter be referred to as the PBBM equation.) Exact time-independent solutions of (45) and (46) are well known; these are the cnoidal waves, of which the solitary wave is a special case. Recently, the $\mathrm{KdV}$ equation has been the subject of extensive research owing to its general applicability in numerous fields in which wave motion occurs in a non-dissipative medium where nonlinearities and frequency dispersion compete. Zabusky \& Kruskal (1965) found numerically that when several solitary waves (or solitons) of different amplitude are localized in a small spatial region of a fluid medium so that they interact in a nonlinear manner, each soliton eventually emerges from the interaction and retains its initial identity. The only effect of the nonlinear interaction is a slight change in phase of the solitons. This remarkable behaviour of a nonlinear physical process has been confirmed analytically by Lax (1968) for two interacting solitons. Gardner et al. (1967) have discovered a nonlinear transformation which reduces the solution of the $\mathrm{KdV}$ equation to solving the linear inverse scattering problem. They demonstrated that for an initial condition $\eta(x ; 0)$ that approaches a constant sufficiently fast as $|x| \rightarrow \infty$, a train of solitons eventually evolve, ordered by amplitude, and followed by a spreading train of oscillatory waves. Zabusky (1968) further stated that if the initial data satisfy

$$
\int_{-\infty}^{\infty} \eta(x ; 0) d x>0,
$$

i.e. the net wave volume is positive, at least one soliton emerges. When the net wave volume is negative, i.e.

$$
\int_{-\infty}^{\infty} \eta(x ; 0) d x<0
$$

it appears that no simple conclusions can be stated for the asymptotic wave behaviour. (In fact, on the basis of experiments conducted for this study, the asymptotic wave behaviour for this case is apparently a function of the initial amplitude distribution.)

These general properties of the solution of the $\mathrm{KdV}$ equation for an arbitrary initial condition (which would also be expected for the solution of the PBBM equation even though no exact solution algorithm for this equation has been found) have direct application to the waves generated by the bed deformations discussed previously. For positive bed displacements a wave system is generated in which the net volume is finite and positive; for negative bed motions the net volume is finite and negative. In order to find an uniformly valid solution for 
wave behaviour resulting from these bed deformations at every position in the downstream region, the following strategy might be used. If the linear theory provides an adequate description of wave behaviour initially, then this theory may be used until $U$ (which is computed in a proper manner) indicates that nonlinearities are becoming important. Once this stage of evolution is reached, the $\mathrm{KdV}$ or PBBM equation is required to describe further wave motion. Computations using the linear theory can be matched to the KdV or PBBM equations by using the linear solution in its region of applicability as the initial condition for either of these equations.

In order to apply the suggested strategy for determining wave behaviour in the downstream region, it is imperative that $U$ be an easily definable quantity and that a numerical value be established which indicates when amplitude and frequency dispersion are of equal importance. For both bed motions investigated herein, the waves entering the downstream region of the fluid domain are complex in form, i.e. non-sinusoidal. A single characteristic length $l$ which adequately describes the entire wave may not exist for waves of this type; hence, the length $l$ becomes a local property of various regions of the wave. An appropriate definition for $l$ in a region of these complex waves is $l=O\left(\eta / \eta_{x}\right)$, where $\eta_{x}$ is the slope of the wave. In order to establish a numerical value for the characteristic length, the operational definition $l=\left|\eta_{0}\right| /\left|\left(\eta_{x}\right)_{\max }\right|$ may be used, where $\eta_{0}$ is the total change in wave amplitude within a region and $\left(\eta_{x}\right)_{\max }$ is the maximum slope of the wave in the region. Using this definition for $l$, the ratio of nonlinear and linear effects becomes

$$
U=\eta_{0}^{2}\left|\eta_{0}\right| / h^{3}\left(\eta_{x}\right)_{\max }^{2}
$$

where the absolute value of $\eta_{0}$ has been used to prevent $U$ from becoming negative. Equation (48) suggests that a complex wave may be divided into regions by the positions of zero slope along the wave form. The numerical value of $U$ as defined by (48) which indicates that amplitude and frequency dispersion are equal can be found from the Boussinesq profile for the solitary wave; applying (48) to either region of this wave yields $U \approx 2$ (to one significant digit). It should be noted that, in judging the applicability of the linear theory for determining complex wave behaviour by the magnitude of $U$, the largest value along the wave profile should be used; generally, the maximum value will occur in the leading region of the wave.

Owing to the complexity of the analytical solution of the $\mathrm{KdV}$ equation for arbitrary initial conditions and the simplicity of the numerical solution of the PBBM equation presented by Peregrine (1966), the latter has been chosen for use in this study. Peregrine found that a simple finite-difference approximation of (46) was stable and that as the non-dimensional grid spacing $\Delta x$ and time step $\Delta t$ were reduced the difference approximation converged to the exact solution. The accuracy of the finite-difference approximation for a specific grid size $\Delta x$ (which was taken equal to $\Delta t$ throughout and will hereafter be referred to as $\Delta$ ) is easily checked by propagating a solitary wave numerically. Since the solitary wave is an exact solution of (46) the wave should propagate unchanged; changes that do occur represent the inaccuracy of the numerical 
model. A convenient measure of this error is the reduction in solitary-wave amplitude during propagation. In the results which follow, $\Delta$ was chosen by first propagating a soliton of amplitude comparable with the wave under consideration and reducing $\Delta$ until the percentage reduction in soliton amplitude over the required distance of propagation was acceptable.

\section{Results in the downstream region}

In order to illustrate the suggested matching technique for finding wave behaviour in the downstream region when the net volume in the wave is positive, an exponential bed displacement with the following generation parameters has been investigated: $\zeta_{0} / h=0 \cdot 1, b / h=12 \cdot 2$ and $t_{c}(g h)^{\frac{1}{2}} / b=0 \cdot 148$. The small timesize ratio and amplitude scale suggest that the linear theory should be applicable initially for this case.

The experimentally determined wave systems entering the downstream region at $(x-b) / h=0$ and at three positions further downstream are shown in figure $10(a)$. Computations for $U$ using (48) in the front region of the wave system are also shown at the downstream positions; all calculations have been rounded to one significant digit. (It should be noted that all experimental measurements are Eulerian, i.e. the temporal distribution of wave amplitude is recorded at a specific location. The spatial structure of these initially linear waves at a time $t$ (say $t=t_{0}=\left(x_{0}-b\right) /(g h)^{\frac{1}{2}}$ ) is equivalent to the temporal record at $x=\left(x_{0}-b\right.$ ) plus an error of order $(h / l)^{2}$; this error is negligible for the waves presented herein. Hence, the front wave slope $\eta_{x}$ of the leading wave is easily determined from figure $10(a)$.) The structure of the wave entering the downstream region at $(x-b) / h=0$ is similar to those shown previously in figure 7 for an impulsive upthrust of the bed; the wave energy is concentrated in an intumescence whose shape resembles the bed deformation. After propagation through 180 depths this intumescence appears to be separating into approximately three waves, ordered by amplitude and followed by a train of small oscillatory waves. This disintegration of the initial intumescence into individual waves continues as shown by measurements at $(x-b) / h=400$, where these waves now resemble solitons near their crest. Computations of $U$ in figure $10(a)$ indicate an initial growth in nonlinear effects until amplitude and frequency dispersion have become about equal. The balance of these two effects appears to be maintained during further propagation. (A similar pattern of wave evolution was also observed in other experiments where the amplitude distribution of the wave system entering the downstream region was varied while maintaining a net positive volume in the wave system.)

Wave profiles computed by the linear theory, i.e. equation (22), for this bed deformation at similar positions in the fluid domain are shown in figure $10(b)$; the Ursell number in the front region of the wave system has also been computed for the downstream positions. Comparison of the computed results at $(x-b) / h=0$ and 20 with the measured profiles in figure $10(a)$ indicates that the linear theory initially provides an adequate description of wave motion. However, during further propagation the linear theory diverges more and more from 


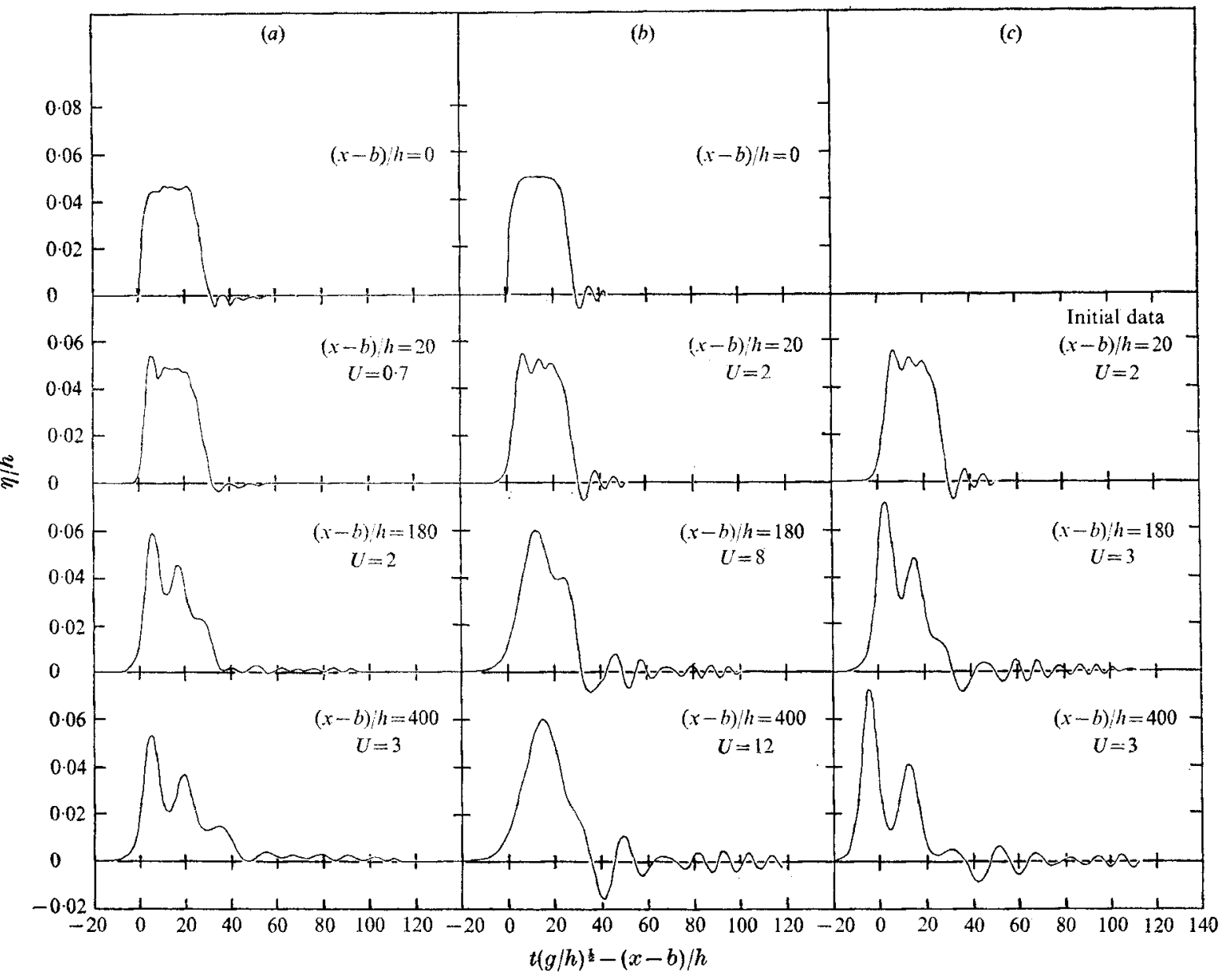

Figure 10. Downstream wave profiles generated by an impulsive exponential bed upthrust; $b / h=12 \cdot 2, \zeta_{0} / h=0 \cdot 1, t_{c}(g h)^{\frac{1}{2}} / b=0 \cdot 148$. (a) Measured. $(b)$ Computed by linear theory. $(c)$ Computed by the PBBM equation using the linear theory at $(x-b) / h=20$ as the initial data.

the measured wave behaviour. This divergence is especially obvious at $(x-b)$ / $h=400$, where the linear theory shows only the disintegration of the initial intumescence into its spectral composition and no soliton formation. Computations of $U$ in figure $10(b)$ demonstrate the continual growth (like $t^{\frac{2}{3}}$ over this range of propagation) of this ratio as the longer wave components appear at the wave front. After only 20 depths of propagation by the linear theory, the Ursell number is seen to equal two; however, as noted previously, the computed and measured wave structures are approximately equivalent up to this position.

Following the suggested technique for determining wave behaviour once nonlinearities become important, the profile computed by the linear theory at $(x-b) / h=20$ has been used as the initial data for the PBBM equation. For the numerical propagation of these initial data, a non-dimensional step size $\Delta$ of $0 \cdot 3$ was used and resulted in a $14 \%$ reduction in amplitude of the test soliton for 
380 depths of propagation. Results of the computations at $(x-b) / h=180$ and 400 are presented in figure $10(c)$. Comparison of the computed wave behaviour with the measurements shown in figure $10(a)$ shows that the PBBM equation produces the same general pattern of wave evolution; solitons are developing and are ordered by amplitude, and a trailing train of oscillatory waves is forming. The ratio of nonlinear to linear effects remains constant during propagation at a value of $U=3$, which indicates that the two effects are about equal. The major discrepancies between the wave behaviour computed by the PBBM equation and the observed wave structure appears in the maximum amplitude and speeds of the evolving waves. At the last station of computation the maximum amplitude of the leading soliton is $40 \%$ larger than that of the measured wave while the average speed of the computed soliton is approximately $3 \%$ greater than the measured speed. Considering a $14 \%$ reduction in the computed amplitude of the leading soliton as a result of the inaccuracy of the numerical model, a difference between theoretical and experimental amplitudes of $60 \%$ is probable. A possible explanation of these differences between theory and experiment is the presence of viscous energy losses and boundary stresses in the experimental measurements; neither of these viscous effects are modelled by the PBBM equation (or by the linear theory). A thorough investigation of the viscous effects on complex wave systems for the wave tank used in this study has not been conducted; however, French (1969) has investigated the viscous decay in amplitude of a single solitary wave propagating in this wave tank and found that the theory of Keulegan (1948) provides an adequate description of this phenomena. (It should be noted that the water depths used by French exceeded the $5 \mathrm{~cm}$ used for the experiment presented in figure $10(a)$.) Although the complex wave system shown in figure $10(a)$ primarily consists of several solitary waves which are localized initially, the application of Keulegan's results to the leading soliton which separates most rapidly from the trailing waves and propagates into a quiescent fluid should yield at least an order-of-magnitude estimate of the viscous damping of this soliton. Using the maximum amplitude of the leading soliton at $(x-b) / h=400$ in figure $10(a)$ as the damped amplitude in Keulegan's results, computations suggest that this measured amplitude would be $57 \%$ larger in the absence of viscosity. Since additional energy losses may also occur in the boundary layer developed at the free surface (see, for example, Van Dorn 1966), which is not considered in Keulegan's analysis, it does appear that viscous effects can account for the large discrepancies between the measured wave amplitudes and those computed by the PBBM equation. (Another potential factor in the discrepancy between theoretical and experimental amplitudes and speeds (as noted by a referee) is the effect of residual vorticity remaining in the wave tank between successive experiments; however, a sufficient period of time was permitted to occur between experiments in order for the residual vorticity to disappear.) Hence, considering the probable effect of viscosity on the experimental measurements, the results presented in figure 10 indicate that the PBBM equation does provide a more satisfactory model of far-field wave behaviour than the linear theory.

In order to illustrate the downstream behaviour of waves resulting from an impulsive downthrow of the bed (thus, when the net volume in the generated 
of the front region of the leading wave results from amplitude dispersion as indicated by $U_{1}$, which has increased to $U_{1}=30$ at $(x-b) / h=180$ and to $U_{1}=55$ at $(x-b) / h=400$. Computations of $U_{2}$ in the rear region of the leading wave indicate that nonlinear and linear effects remain approximately equal during propagation; however, the train of oscillatory waves trailing behind the leading wave continues to spread. No solitons appear to be developing for this particular intumescence where $\eta \leqslant 0$ almost everywhere at $x / h=b / h$. (However, it should be noted that solitons did appear to evolve in other experiments where the net wave volume was negative but the initial wave system entering the downstream region was not negative everywhere.)

The wave profiles computed by the linear theory for this bed deformation at corresponding positions in the fluid domain are shown in figure $11(b)$. The linear theory and measured results agree well initially and for twenty depths of propagation. During further propagation the linear theory begins to deviate from the observed wave structure; this is especially obvious near the front of the leading wave, where nonlinear effects appear to be dominating actual wave behaviour.

In order to determine the applicability of the suggested strategy for using the PBBM equation for this bed displacement, the wave profile computed by the linear theory at $(x-b) / h=20$ has been used as the initial data and propagated numerically $(\Delta=0 \cdot 3)$ to the downstream positions. Results of these computations are shown in figure $11(c)$. Comparison of the computed wave profiles with the observed results in figure $11(a)$ indicates that the general pattern of wave evolution is modelled satisfactorily by the PBBM equation. The major difference in the results again occurs in the predicted wave amplitudes and speeds and may largely be attributable to viscous effects in the experimental model. The stretching of the front portion of the leading wave appears to be modelled well as indicated by the growth of $U_{1}$ from $U_{1}=25$ at $(x-b) / h=180$ to $U_{1}=61$ at $(x-b) / h=400$ (compared with $U_{1}=30$ and 55 at corresponding positions for the experimentally determined profiles). The continued accuracy of the PBBM equation in modelling wave behaviour in this region is questionable since the PBBM equation is derived under the basic assumption that nonlinear and linear effects are about equal; this assumption becomes less valid with time in the front region of the wave system. The computed results indicate that nonlinear and linear effects remain about equal during propagation in the trailing region of the leading wave and similar to the observed behaviour in figure $11(a)$.

The emphasis in this discussion of wave propagation from the source region of a tsunami has been on the gradual breakdown of the linear theory and the importance of the interaction of nonlinear and linear effects in determining far-field wave signatures. For this model of tsunami generation it appears that the PBBM equation provides a satisfactory model of wave behaviour when the bed deformation is such that the net wave volume is finite and positive. For the particular bed deformation investigated where the net volume of the generated wave was negative and the amplitude of the initial wave system was negative almost everywhere, the PBBM equation appears to be a satisfactory model during the observed period of propagation: however, its continued applicability 
near the front region of the leading negative wave is questionable. The extrapolation of conclusions based on this simple model of wave propagation to prototype phenomena is severely limited by the assumptions of uniform depth and a two-dimensional fluid domain. Most tsunamigenic earthquakes occur in the shallow water around the continental shelves of the oceans; hence the generated wave soon propagates across the continental slope into the deep ocean. The increasing depth would tend to delay the growth of nonlinear effects thus making the linear theory valid for longer times than observed in this study. The threedimensional spreading of the wave energy in an actual tsunami at large distances from the source region would also tend to reduce or in some cases completely negate the growth of nonlinear effects. (It should be noted that a two-dimensional model of wave propagation may be valid initially since typical source regions of tsunamis appeared to be strongly elongated along the principal fault line.)

The author is greatly indebted to Professor Fredric Raichlen for his kind guidance through every phase of this study. Gratitude is also extended to other members of the faculty and staff of the W.M. Keck Laboratory of Hydraulics and Water Resources, California Institute of Technology. This work was supported by the National Science Foundation under Grants GK-2370 and GK24716 .

\section{REFERENCES}

Benjamin, T. B., Bona, J. L. \& Mahony, J. J. 1972 Model equations for long waves in nonlinear dispersive systems. Phil. Trans. Roy. Soc. 272, 47-78.

French, J.A. 1969 Wave uplift pressures on horizontal platforms. W. M. Keck Lab. Hydraul. \& Water Res. Calif. Inst. Tech. Rep. KH-R-19.

Gardner, C. S., Greene, J. M., Kruskal, M. D. \& Miura, R. M. 1967 Method for solving the Korteweg-de Vries equation. Phys. Rev. Lett. 19, 1095-1097.

Hammack, J.L. 1972 Tsunamis: a model of their generation and propagation. $W . M$. Keck Lab. Hydraul. \& Water Res., Calif. Inst. Tech. Rep. KH-R-28.

Honda, H. \& Nakamura, K. 1951 The waves caused by one-dimensional deformation of the bottom of shallow sea of uniform depth. Sci. Rep. Tohoku University, Sendai, Japan, 3, 133-137.

Hwang, L. S. \& Drvoky, D. 1970 Tsunami generation. J. Geophys. Res. 75, 6802-6817.

ICHIYE, T. 1950 On the theory of tsunami. Oceanograph. Mag. 2, 83-100.

ICHIYe, T. 1958 A theory of the generation of tsunami by an impulse at the sea bottom. J. Oceanograph. Soc. Japan, 14, 41-44.

JefFreys, H. \& Jeffreys, B. S. 1946 Methods of Mathematical Physics, 1st edn. Cambridge University Press.

KaJturA, K. 1963 The leading wave of a tsunami. Bull. Earthquake Res. Inst. Tokyo University, 41, 535-571.

KELler, J. B. 1963 Tsunamis: water waves produced by earthquakes. Int. Un. Geodesy de Geophys. Monograph, no. 24, pp. 154-166.

KeUlegan, G. H. 1948 Gradual damping of solitary waves. J. Res. Nat. Bur. Stand. 40, 487-498.

KorteweG, D. J. \& De VRIES, G. 1895 On the change of form of long waves advancing in a rectangular canal, and on a new type of long stationary waves. Phil. Mag. 39 (5), 422-443.

LAx, P. D. 1968 Integrals of nonlinear equations of evolution and solitary waves. Comm. Pure \& Appl. Math. 21, 457-490. 
Mryer, R. E. 1967 Note on the undular jump. J. Fluid Mech. 28, 209-221.

Momor, T. 1964 Tsunami in the vicinity of a wave origin. Bull. Earthquake Res. Inst. Tokyo University, 42, 133-146.

NakamuRA, K. 1953 On the waves caused by the deformation of the bottom of the sea: I. Sci. Rep. Tohoku University, Sendai, Japan, 5 (5), 167-176.

Peregrine, D. H. 1966 Calculations of the development of an undular bore. J. Fluid Mech. 25, 321-330.

Plafker, G. 1969 Tectonics of the March 27, 1964 Alaska earthquake. Geol. Survey Prof. Paper, no. 543-1.

STokes, G. G. 1847 On the theory of oscillatory waves. Trans. Camb. Phil. Soc. 8, 441455.

Takahasi, R. 1963 On some model experiments on tsunami generation. Int. Un. Geodesy \& Geophys. Monograph, 24, pp. 235-248.

TAkahasi, R. \& Hatori, 'T. 1962 A model experiment on the tsunami generation from a bottom deformation area of elliptic shape. Bull. Earthquake Res. Inst. Tokyo University, 40, 873-883.

Tuck, E. O. \& HwaNG, L. S. 1972 Long wave generation on a sloping beach. J. Fluid Mech. 51, 449-461.

Urseli, F. 1953 The long-wave paradox in the theory of gravity waves. Proc. Camb. Phil. Soc. 49, 685-694.

VAN Dorx, W. G. 1964 Source mechanism of the tsunami of March 28, 1964 in Alaska. Proc. 9th Conf. Coastal Engng, Lisbon, pp. 166-190.

VAN Dons, W. G. 1966 Boundary dissipation of oscillatory waves. J. Fluid Mech. 24, $769-779$.

WeBB, L. M. 1962 Theory of waves generated by surface and sea-bed disturbances. In The Nature of Tsunamis, Their Generation and Dispersion in Water of Finite Depth, Tech. Rep. no. SN 57-2. Nat. Engng. Sci. Co.

ZABUSkx, N.J. 1968 Solitons and bound states of the time-independent Sehrödinger equation. Phys. Rev. 168, 124-128.

ZABUSKY, N.J. \& KRUSKAL, M. D. 1965 Interactions of 'solitons' in a collisionless plasma and the recurrence of initial states. Phys. Rev. Lett. 15, 240-243. 
Journal of Fluid Mechanics, Vol. 60, part 4

Plate 1

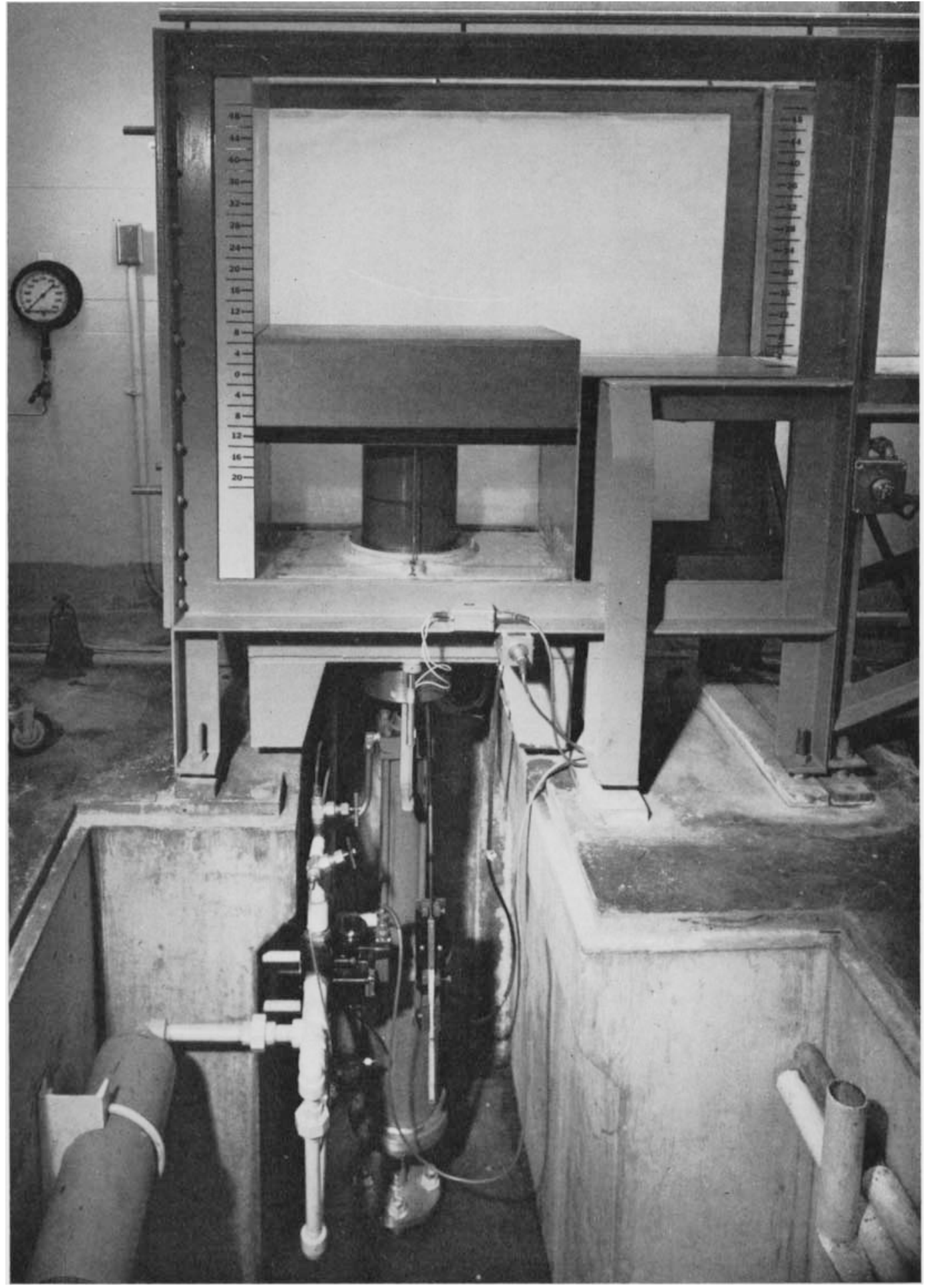

